\title{
Conceptual Realism Versus Quine on Classes and Higher-Order Logic
}

\author{
Nino B. Cocchiarella
}

\begin{abstract}
The problematic features of Quine's 'set' theories NF and ML are a result of his replacing the higher-order predicate logic of type theory by a first-order logic of membership, and can be resolved by returning to a second-order logic of predication with nominalized predicates as abstract singular terms. We adopt a modified Fregean position called conceptual realism in which the concepts (unsaturated cognitive structures) that predicates stand for are distinguished from the extensions (or intensions) that their nominalizations denote as singular terms. We argue against Quine's view that predicate quantifiers can be given a referential interpretation only if the entities predicates stand for on such an interpretation are the same as the classes (assuming extensionality) that nominalized predicates denote as singular terms. Quine's alternative of giving predicate quantifiers only a substitutional interpretation is compared with a constructive version of conceptual realism, which with a logic of nominalized predicates is compared with Quine's description of conceptualism as a ramified theory of classes. We argue against Quine's implicit assumption that conceptualism cannot account for impredicative concept-formation and compare holistic conceptual realism with Quine's class Platonism.
\end{abstract}

Does logic include set theory, or does logic leave off where set theory begins? That is a question that Quine has often asked, and his answer has not always been the same. In his early work, for example, Quine's answer was that set theory was indeed part of logic, but that was because set theory was really a "cleaned up" version of higher-order logic. ${ }^{1}$ In his later work, on the other hand, certainly at least since the Philosophy of Logic, Quine's

\footnotetext{
${ }^{1}$ CF. Hahn and Schilpp 1986, p. 10.
} 
answer has been that set theory is not part of logic, and that is because there really is no such thing as higher-order, as opposed to first-order, logic. A fundamental assumption underlying his position in both his early and his later work is that set theory is part of logic if, and only if, logic can be divided into an elementary, first-order part and a nonelementary, "higher-order" part. Indeed, Quine has continuously maintained that if there is a nonelementary, "higher-order" part to logic, then it is the same as set theory. Thus, in his 1954 essay, 'Carnap and Logical Truth,' Quine writes that after elementary, first-order logic "[t]he further part of logic is set theory, which requires that there be classes among the values of its variables of quantification" (Quine 1966, p. 103). More specifically, "higher-order" logic, according to Quine, is just one or another version of first-order set theory, and to limit logic to its elementary, first-order part "is merely to deprive ' $\in$ ' of the status of a logical word" (ibid., p. 104). It is only because "the contrasts between elementary logic and set theory are so fundamental," Quine has maintained, "that one might well limit the word 'logic' to the former (though I shall not) and speak of set theory as mathematics in a sense exclusive of logic" (ibid.; italics added). In time, Quine did make just this move, and he has maintained that position ever since.

But is higher-order logic the same as one or another version of set theory? Or, as Quine would have preferred it in his early works, is set theory really just a 'cleaned up' version of higher-order logic? And, is set theory part of logic if, and only if, logic can be divided into an elementary, first-order part and a nonelementary 'higher-order' part? To the first question, even Quine would now say, 'No' - or, rather, he would reject it altogether, because he now maintains that logic is not to be divided into an elementary part and a higher-order part. But, to the second question, he must say, 'Yes,' because that is an assumption that he has been implicitly committed to throughout his career. In what follows, we will not challenge Quine's view that set theory is not part of logic (and, in fact, we agree with it), but we will reject his implicit assumption that set theory is part of logic if, and only if, logic can be divided into a first- and a higher-order part. In particular, we will argue that, unlike his view of set theory today, which accords with the iterative concept of set, Quine's early views on set theory - i.e., the views he developed in his systems NF (of his 1936 paper, 'New Foundations for Mathematical Logic') and ML (of his 1940 book, Mathematical Logic) - are really not about sets but about classes in the logical sense, i.e., classes as the extensions of concepts, and, consequently, that he is implicitly committed in 
his early works to a framework of concepts that can be couched only in a higher-order logic of predication. We assume in this regard that sets are not classes, by which we mean only classes in the logical sense, i.e., classes as the extensions of concepts. For, whereas classes have their being in the concepts whose extensions they are, sets have their being in their members, a fact that is reflected in the iterative process by which they are formed. That is why there is a universal class but no universal set, and also why classes, but not sets, have absolute complements. Both classes and sets have an axiom of extensionality as a criterion of identity, but only sets are subject to an axiom of foundation, which, as an essential part of the iterative process by which they are formed, is no less essential to their individuation. Only classes, on the other hand, are subject to Russell's paradox, which is inapplicable to sets because it assumes a pattern of set-formation that does not accord with the iterative process by which sets are formed. Whatever Quine's reasons for having 'plumped for' the iterative concept of set in his later works, we agree with his claim that set theory is not part of logic, higher-order or. otherwise, but we reject the basis on which he makes that claim, namely, that there is no such thing as higher-order, as opposed to first-order, logic.

In this regard, we will first argue against Quine's replacement of a theory of predication by a theory of membership simpliciter. We will show, in particular, how most of the problematic features of Quine's 'set' theories NF and ML are easily explained once those theories are seen in their proper context as part of a second-order logic of predication. ${ }^{2}$ We then argue against Quine's claim that bound predicate variables can only have as values the objects that nominalized predicates denote as abstract singular terms. We will explain, in particular; how Quine's use of Russell's paradox in a second-order logic with nominalized predicates as abstract singular terms is fallacious, and how we can even add an unstratified, impredicative comprehension principle to the logic in question without generating any inconsistency. Then, in terms of our own interpretation of the distinction between the concepts that predicates stand for in their role as predicates and the objects that nominalized predicates denote in their role as abstract singular terms, we argue against Quine's way of distinguishing nominalism from conceptualism, and conceptualism from Platonism. We call the general framework of our interpretation of this distinction conceptual realism, and we argue that, contrary to Quine's

\footnotetext{
${ }^{2}$ We will use 'set' with scare-quotes to indicate that, Quine's intentions notwithstanding, the entities in question are really not sets but classes in the logical sense.
} 
view of conceptualism, this framework is not restricted to a ramified predicative logic. In terms of what we call holistic conceptual realism, we conclude with a defense of not one but two alternative versions of higher-order logic as a second-order logic with nominalized predicates as abstract singular terms. Both are impredicative logics, and, in that regard, both contain, either directly or indirectly, an impredicative theory of classes in the logical sense, i.e., as the extensions of concepts. Thus, even though logic does not include set theory, it does contain, either directly or indirectly, an impredicative theory of classes.

\section{Predication Versus Membership}

According to Quine, in one of his later works, the pioneers in modern logic, such as Frege and Russell, overestimated the kinship between membership and predication and in that way came to view set theory as logic (Quine 1970, p. 65). Such a claim, we maintain, is both false and misleading. Frege and Russell did assume a logical kinship between predication and membership, but what they meant by membership was membership in a class as the extension of a concept (where a concept is a predicable entity, i.e., a universal in the traditional sense) and not membership in a set. Sets, unlike classes, as we have said, have their being in their members, and in that regard there need be no kinship at all between predication and membership in a set. Classes in the logical sense, on the other hand, have their being in the concepts whose extensions they are, which means that any theory of membership in a class presupposes a superseding theory of predication. ${ }^{3}$ Frege and Russell did not view set theory as logic, but they each did develop a theory of classes and they each did so based on a superseding higher-order theory of predication.

Quine rejects the distinction between sets and classes in the logical sense. He does so because he rejects the whole idea of there being predicable entities such as concepts (or properties, or propositional functions, etc.) that are different from the classes that are their extensions. That is why it was he, and not Frege or Russell, who came to view set theory as logic in his early work; and he did so by overestimating the kinship between membership and predication in the most extreme way, namely, by identifying them. Thus, after noting that predication was taken as a primitive of his 1932 dissertation, which he describes as a "cleaning up" of Russell and Whitehead's Principia

\footnotetext{
${ }^{3}$ Cp. Frege 1979, p. 183.
} 
Mathematica, Quine goes on to say that because his logic was extensional "predication was membership" (Hahn and Schilpp 1986, p. 10). But Frege's logic was also extensional, and it would be quite wrong to say that for him predication was membership. Indeed, Frege is emphatic in maintaining that a "concept is logically prior to its extension" and that we should regard "as futile the attempt to take the extension of a concept as a class, and make it rest, not on the concept, but on single things" (Frege 1952, p. 106), which in effect would be to replace the logical notion of a class with the mathematical notion of a set.

After 'cleaning up' Principia in his dissertation, which he later revised and published as System of Logistic (1934), Quine went on to develop his 'set' theory NF of "New Foundations," which he describes as a first-order reconstruction of the theory of simple types. On Quine's interpretation, the real ontological import of the logico-grammatical hierarchy of the theory of simple types resides in the constraints it imposes on the conditions for positing 'sets', namely, that those conditions must be stratified with respect to the membership relation. ${ }^{4}$ Thus, the comprehension principle for positing 'sets' in NF as a first-order logic (with ' $\in$ ' as its only primitive predicate constant) is given as follows:

If $\varphi$ is a stratified formula of $\mathbf{N F}$ in which $y$ does not occur free, then $(\exists y)(\forall x)(x \in y \leftrightarrow \varphi)$ is a theorem of $\mathbf{N F}$.

This principle, as Quine observes, is to be understood as a replacement of the original "naive" comprehension principle,

$$
(\exists y)(\forall x)(x \in y \leftrightarrow \varphi),
$$

where $\varphi$ is any formula, stratified or not, in which $y$ does not occur free. The "naive" principle must be replaced, of course, because, by putting $x \notin x$ for $\varphi$, it yields Russell's paradox.

The naive comprehension principle, it should be noted, is really about membership in a class in the logical sense. Indeed, it is really a schematic version of a second-order principle to the effect that every concept has an extension:

$$
(\forall F)(\exists y)(x \in y \leftrightarrow F(x)) .
$$

\footnotetext{
${ }^{4} \mathrm{~A}$ first-order formula $\varphi$ with ' $\in$ ' as its only predicate constant is stratified if there is an assignment $t$ of natural numbers to the variables occurring in $\varphi$ such that for all variables $u, v$, if ' $u \in v$ ' occurs in $\varphi$, then $t(v)=t(u)+1$.
} 
It is this second-order principle that expresses the logical kinship between predication and membership, which, according to Quine, Frege and Russell overestimated and which led them to see set theory as logic. Frege and Russell did assume the second-order principle, and in fact it is easily seen to be provable in the system of Frege's Grundgesetze, i.e., Frege's second-order logic with abstracts as singular terms denoting value-ranges (Wertverläufe). But, contrary to Quine's claim, this does not mean that either Frege or Russell was led to see set theory as logic, or even as a part of logic: for the classes in question were not sets but classes as the extensions of concepts. Frege and Russell were not concerned with set theory, but with a logic of concepts (including relational concepts), and only thereby, derivatively, also with a logic of the classes that are the extensions of concepts.

Russell's paradox really has two forms, one in regard to membership in a class in the logical sense, which, as already explained, presupposes a superseding theory of predication, and the other in regard to predication, dealing, as Russell put it in his 1902 letter to Frege, with "the predicate: to be a predicate that cannot be predicated of itself" (Frege 1980, p. 130). (Russell, in the period in question, took 'predicate' to be synonymous with 'concept'.) Russell himself came to avoid the membership form of his paradox by adopting his famous "no classes" theory, according to which all talk of classes is reducible to talk of the concepts whose extensions they are. This theory provides perhaps the strongest interpretation of what it can mean to say that classes have their being in the concepts whose extensions they are. It also assumes that concepts themselves are objects, contrary to Frege's view of them as ungesättigt, i.e., as unsaturated predicable entities, which does lead to some confusion in Russell's early views on the role of concepts in predication. ${ }^{5}$ Be that as it may, Russell then came to avoid the predication form of his paradox by adopting type-theoretical restrictions that made it meaningless on grammatical grounds alone to predicate a concept of itself, i.e., to apply a predicate to a nominalized form of itself as an abstract singular term.

Quine, quite properly, rejected the grammatical restrictions Russell imposed on his theory of predication in order to avoid his paradox. The real import of those restrictions was not meaningfulness but ontology. That is, Russell's type-theoretical constraints should be taken as conditions for posit-

\footnotetext{
${ }^{5}$ For a discussion of some of the difficulties in Russell's view of the role of concepts in predication, see Cocchiarella (1980).
} 
ing concepts as abstract entities and not as conditions for the meaningful application of predicates to nominalized forms of predicates as abstract singular terms. Thus, instead of Russell's grammatical division of predicates into different logical types as a way to avoid his paradox, Quine proposed that we should reformulate the comprehension principle for positing concepts by requiring only that the formulas in question should all be stratified.

The idea of turning to a stratified comprehension principle for the positing of concepts was an important insight, and, as I have explained elsewhere, it has a motivation that can be based on the original source of Russell's ideas about type theory: namely, Frege's hierarchy of concepts and what I have called Frege's double-correlation thesis mapping higher-level concepts into first-level concepts and the latter into their extensions. ${ }^{6}$ In this regard, Quine was right to insist on replacing Russell's grammatical restrictions about the conditions under which predicates can be applied to predicates (or, rather, to nominalized forms of the latter) by a stratified comprehension principle. But, he was wrong in making the additional move of identifying predication with membership and concepts with their extensions, thereby eliminating not only Russell's division of predicates into different logical types but also, and more importantly, the distinction between predicates and singular terms as well. By identifying predication with membership, Quine replaced what should have been a second-order stratified comprehension principle about predication, including the way it applies to nominalized predicates as abstract singular terms, by a first-order stratified comprehension principle about membership. In this way, Quine rejected the whole idea of predication as something different from membership and replaced Russell's higher-order theory of predication by a first-order theory of membership, which he has continued to describe as a version of set theory, but which he no longer considers to be part of logic.

\section{Old Versus New Foundations}

The idea of a stratified comprehension principle for second-order predicate logic with nominalized predicates as abstract singular terms is a return to old foundations of logic, as opposed to Quine's new foundations; that is, it is a return to foundations based on a theory of predication instead of a theory of membership. In its simplest form, the idea is to modify Frege's original

\footnotetext{
${ }^{6}$ Cf. Cocchiarella 1985b and 1986c.
} 
second-order predicate logic (as described in his Begriffsschrift) by allowing nominalized predicates to occur as singular terms on a par with individual variables. This is a somewhat simpler version than Frege's own extension of his logic in his Grundgesetze where predicate nominalizations are represented by the application of his smooth-breathing abstraction operator to open formulas. ${ }^{7}$ In this simpler version, to nominalize a predicate, which we assume has parentheses (and commas, in the case of a relational predicate) surrounding its subject-positions, we need simply delete the parentheses (and commas) that are an integral part of that predicate in its role as a predicate. Thus, for example, in the following analysis of membership in terms of predication,

$$
x \in y=_{d f}(\exists F)[y=F \wedge F(x)],
$$

we have a predicate letter occurring in the definiens both in its role as a predicate (with its accompanying pair of parentheses) and (without its accompanying pair of parentheses) as an abstract singular term, i.e., as a singular term on a par with individual variables. ${ }^{8}$ On this analysis, given Russell's view of concepts as having an individual as well as a predicable nature, we have another version of Russell's "no classes" theory, according to which all talk of classes is explained in terms of talk of concepts. Actually, even aside from Russell's "no classes" theory, this analysis of membership corresponds very closely to Frege's own definition (as represented by the function $\xi \cap \zeta$ given in Volume 1, sec. 34, of his Grundgesetze). That is, given Frege's view of concepts as unsaturated predicable entities (as opposed to Russell's view of concepts as higher-order objects), as well as his view of nominalized predicates as denoting the extensions of the concepts that those predicates otherwise stand for in their role as predicates, the above analysis of membership also expresses Frege's view of what it means for a class to have its being in the concept whose extension it is.

Before stating the second-order stratified comprehension principle for concepts, we should note that, because relational predication is also taken primitively, we. must require that the formulas occurring in such a principle must

\footnotetext{
${ }^{7}$ Cf. Cocchiarella (1986c, sec. 4) for a detailed defense of the claim that Frege's abstracts for value-ranges amounted to his logical treatment of nominalized predicates as abstract singular terms.

${ }^{8}$ This means that in addition to $(\forall y) \varphi \rightarrow \psi(x / y)$ as an instance of the first-order universal instantiation law, we also have $(\forall y) \varphi \rightarrow \varphi(F / y)$. For the formal details of these matters, see Cocchiarella (1986a, chap. 4).
} 
be not merely stratified but homogeneously stratified. ${ }^{9}$ Otherwise, predication, which is specifiable by means of a heterogeneously-stratified formula, will itself be construed as a relation, in which case Russell's paradox will again be provable. Thus, in this reconstruction of our old foundations for logic, which we call $\mathbf{H S T}^{*}$ (for homogeneous simple types as a second-order logic with nominalized predicates as abstract singular terms), the stratified comprehension principle for positing concepts can now be stated as follows (for all positive integers $n$ ):

$\left(\mathbf{H S C P}^{*}\right)$ : If $\varphi$ is a homogeneously-stratified formula in which $F$ does not occur free, then $(\exists F)\left(\forall x_{1}\right) \ldots\left(\forall x_{n}\right)\left[F\left(x_{1}, \ldots, x_{n}\right) \leftrightarrow \varphi\right]$ is a theorem of $\mathbf{H S T}^{*}$.

Note that because all formulas of standard second-order logic (i.e., formulas having no nominalized predicates occurring as singular terms) are homogeneously stratified, $\mathbf{H S T}^{*}$ includes all instances of the comprehension principle of standard second-order logic among its theorems; and in fact it contains standard second-order predicate logic as a proper part of itself. ${ }^{10}$ Given the above analysis of membership, HST* also contains Quine's stratified comprehension principle for membership, which, along with the reasons already given, is why we say that the classes of $\mathbf{N F}$ are really classes in the logical sense.

Of course, Quine also assumed the extensionality axiom,

$$
(\forall y)(\forall z)[(\forall x)(x \in y \leftrightarrow x \in z) \rightarrow y=z],
$$

as a basic law of logic (that is, of NF when he took it to be the nonelementary part of logic), and (ext) is not provable in $\mathbf{H S T}^{*}$. Stated in this unqualified way, the extensionality axiom, (ext), has some rather strange and undesirable consequences in $\mathbf{N F}$, which, significantly, are not provable in $\mathbf{H S T}^{*}$ even when an extensionality axiom is added to the latter. One problem with (ext), for example, is that it leaves no way in NF by which to distinguish

\footnotetext{
${ }^{9} \mathrm{~A}$ formula $\varphi$, based on predication rather than membership, is homogeneously stratified if there is an assignment $t$ of natural numbers to both the predicate and singular terms occurring in $\varphi$ such that for each atomic subformula $R\left(a_{1}, \ldots, a_{n}\right)$ of $\varphi,(1) t\left(a_{i}\right)=t\left(a_{j}\right)$, for all $i, j$ such that $1 \leq i, j \leq n$, and $(2) t(R)=t\left(a_{1}\right)+1$. (Also, in case identity is taken as a primitive logical constant instead of being defined as indiscernibility, then $t(a)=t(b)$ for all identity formulas $(a=b)$ occurring as part of $\varphi$.)

${ }^{10}$ For the formal details in the description of $\mathbf{H S T}^{*}$, see Cocchiarella (1986a, chap. 4).
} 
concrete objects (i.e., non-classes) from the empty class, because both the empty class and any object that is not a class are memberless and, therefore, vacuously have the same members. To resolve this problem, Quine proposed that non-classes can be identified with their singletons and, therefore, that they can be construed as classes after all. Alternatively, Quine could have introduced another primitive predicate into NF by which to distinguish classes from non-classes. For example, Quine could have taken 'Class' as a primitive predicate constant and assumed

$$
(\forall y)(\forall z)[\operatorname{Class}(y) \wedge \operatorname{Class}(z) \wedge(\forall x)(x \in y \leftrightarrow x \in z) \rightarrow y=z)
$$

instead of (ext), in which case it would not follow that all non-classes are identical with the empty class.

Quine, apparently, was reluctant to introduce any descriptive predicate constant into $\mathbf{N F}$ as a primitive of what he then viewed as the nonelementary part of logic. For the difference between logic and its applications is that logic includes no primitive descriptive constants as a proper part of itself, which is why ' $\in$ ', taken as a replacement for predication, was originally construed by Quine as a logical constant. Of course, had he left predication intact and adopted a second-order logic with nominalized predicates as abstract singular terms, the axiom of extensionality (in the monadic case) would have been formulated simply as:

$\left(\mathbf{E x t}^{*}\right) \quad(\forall F)(\forall G)[(\forall x)(F(x) \leftrightarrow G(x)) \rightarrow F=G]$,

in which case, because of the fundamental logical distinction between predicates and singular terms, there is no need to introduce a descriptive predicate to mark the distinction between concepts and objects. Nor, of course, is there any need to construe all individuals as classes, such as Quine proposed in his defense of (ext) as a basic law of logic.

Quine's proposal to construe all individuals as classes can be represented in monadic $\mathbf{H S T}^{*}$ as follows:

$$
(\forall x)(\exists F)(x=F) .
$$

We do not suggest that we take this proposal seriously; but it is noteworthy that on the basis of our Fregean analysis of membership every theorem of Quine's so-called 'set' theory $\mathbf{N F}$ is a theorem of monadic $\mathbf{H S T} \mathbf{T}^{*}+\left(\mathbf{E x t} \mathbf{t}^{*}\right)+\left(\mathbf{Q}^{*}\right)$, 
which again justifies our claim that the classes of $\mathbf{N F}$ are not sets but classes in the logical sense. We might also note that, by misconstruing predicates as singular terms and identifying predication with membership, every theorem of monadic $\mathbf{H S T} \mathbf{T}^{*}+\left(\mathbf{E x t}^{*}\right)+\left(\mathbf{Q}^{*}\right)$ is translated into a theorem of $\mathbf{N F}{ }^{11}$

Quine's thesis, $\left(\mathbf{Q}^{*}\right)$, is provably equivalent in monadic $\left.\mathbf{H S T} \mathbf{S T}^{*}+\mathbf{E x t}^{*}\right)$ to the extensionality axiom (ext); that is,

$$
\left(\mathbf{E x t}^{*}\right) \vdash_{H S T^{*}}\left[\left(\mathbf{Q}^{*}\right) \leftrightarrow(\mathbf{e x t})\right]
$$

Therefore, from the perspective of our old foundations, as represented by HST*, whatever problems Quine has with his extensionality axiom, (ext), in his new foundations are really the result of his proposal to construe all individuals as classes. One such problem in particular is the fact that on the basis of (ext) as an axiom of NF, Ernst Specker (1953) was able to show that the axiom of choice is refutable in $\mathbf{N F}$ and, therefore, that there must exist infinite classes in NF (since the axiom of choice is provable for finite classes). The axiom of choice is compatible with the theory of types, however, and not refutable in it; and the axiom. of infinity, which in the theory of types is taken as a contingent matter (about the number of concrete individuals in the world), is certainly not provable in that framework other than on the basis of its own assumption. Clearly, something has gone wrong with Quine's reconstruction of the theory of types as a first-order theory of membership.

What has gone wrong, apparently, is Quine's identification of predication with membership, and of concepts with their extensions. Indeed, as is shown elsewhere (in Cocchiarella 1985b), the axiom of choice is not refutable in $\mathbf{H S T}^{*}+\left(\mathbf{E x t}^{*}\right)$, nor is it provable (except by assuming additional axioms) that there are any infinite classes. In fact, what can be proved is that HST $^{*}+\left(\right.$ Ext $\left.^{*}\right)$ is equiconsistent with the theory of simple types (when extensionality axioms are added to the latter). ${ }^{12}$ Thus, it is the return to old foundations about predication, not Quine's new foundations about membership, that is the proper way to view his insight about replacing Russell's grammatical restrictions by a stratified comprehension principle. Also, from this perspective, it is easily seen how it is Quine's proposal to construe all individuals as classes (or concept-correlates) that leads to the problematic results that Specker has proved about NF.

\footnotetext{
${ }^{11}$ Cf. Cocchiarella (1986a, chap. 4, sec. 9) for more details of this relationship between $\mathbf{N F}$ and $\mathbf{H S T}^{*}+\left(\mathbf{E x t}^{*}\right)+\left(\mathbf{Q}^{*}\right)$.

${ }^{12}$ See Cocchiarella 1985b, sec. 6 .
} 
Finally, we might note that the second-order principle that expresses the logical kinship between predication and membership, i.e.,

$$
(\forall F)(\exists y)(\forall x)(x \in y \leftrightarrow F(x)),
$$

is not only consistent but is in fact provable in $\mathbf{H S T}^{*}$ on the basis of the Fregean analysis of membership given above. Indeed, it is precisely on the basis of this principle, together with the homogeneously-stratified comprehension principle for concepts, that we are able to derive Quine's stratified comprehension principle for classes as a theorem schema of $\mathbf{H S T}^{*}$. Thus, it is only on the basis of the logical kinship between predication and membership in a class as the extension of a concept that Quine's principle, as a thesis about the conditions under which classes can be said to exist, can be validated. From the perspective of $\mathbf{H S T}^{*}$ as a way of returning to our old foundations for logic, in other words, Frege and Russell did not 'overestimate' the kinship between predication and membership but had it exactly right.

\section{Concepts Versus Ultimate Classes}

Quine's problem with concepts, or attributes, or any other candidate for a predicable entity, is that we seem to have no adequate principle of individuation for them. Indeed, Quine's recurring dictum is that "there is no entity without identity" (cf. 'On the Individuation of Attributes,' in Quine 1981). Of course, by identity Quine means a relation between objects, and, as in Frege's logic, there are good reasons not to think of concepts as objects. In responding to such an objection, Quine wonders "whether we might just acquiesce in the faulty individuation of attributes ... by treating them as twilight entities, only real enough to be talked of in a few limited contexts, excluding the identity context" (Quine 1981, p. 107).

What Quine proposes as a way of dealing with concepts or attributes as twilight entities is that we should construe them as "ultimate" classes and, in particular, as the ultimate classes that he introduced in the development of his 1940 system ML. For the ultimate classes of ML are like concepts or attributes as naively considered in that they are specified in ML through an unqualified, impredicative comprehension principle, and for that reason, given Russell's paradox, they also could have been said by Quine to have a shadowy existence, or faulty individuation-though in fact Quine does not speak of them this way. In $\mathbf{M L}$, which contains all of the classes of $\mathbf{N F}$ 
but goes beyond them in containing ultimate (or proper) classes as well, the classes that do not have an individuation problem are called 'sets', by which Quine now means any class that is a member:

$$
\operatorname{Set}(x){ }_{d f}(\exists z)(x \in z) .
$$

That is, to be a member, on this construal of Quine, is the same as to be an object whose individuation is not in question. In NF all classes are 'sets' in this sense, because in NF every object belongs at least to its singleton, as well as to many other classes. The ultimate classes of ML, on the other hand, although they are objects in the sense of being values of bound individual variables, are classes that are not members of any other classes and, therefore, like concepts or attributes, their individuation should also be said to be faulty, or at least in question (though Quine, as we have said, does not speak of them in this way). Of course, the 'individuation' of concepts as unsaturated predicable entities is problematic only because concepts are not objects to begin with (i.e., they are not values of bound individual variables), whereas ultimate classes, in order to be objects (that is, in order to be values of bound individual variables), must be individuated. The question of the 'individuation' of concepts is a pseudo-problem, in other words, arising from the confusion of concepts with objects, whereas the individuation of ultimate classes is not a pseudo-problem precisely because they are objects, or at least they are construed as such in ML. But, then, if ultimate classes really do have an individuation problem as objects, as we maintain they do (even if Quine does not), why bring them in at all?

Quine's original reason for adding ultimate classes to the 'sets' of NF was because the principle of mathematical induction,

$$
\varphi(0) \wedge(\forall x)[N(x) \wedge \varphi(x) \rightarrow \varphi(x+1)] \rightarrow(\forall x)[N(x) \rightarrow \varphi(x)],
$$

is provable in NF only when the formula $\varphi$ is stratified. Here, the notion of a natural number (as represented by the predicate $N$ ) is essentially Frege's, since that is the notion that is both natural and appropriate in NF (and that is because $\mathbf{N F}$ is really a theory of classes in the logical sense). As defined in both NF and ML, in other words, a natural number is any class that belongs to every class (ultimate or otherwise) to which 0 belongs and which is closed under the successor relation. By definition, accordingly, the only form of mathematical induction that can be proved in $\mathbf{N F}$ is one that is restricted to stratified formulas (even though not all of the formulas of NF are stratified), 
because, on the basis of the stratified comprehension principle, it is only those formulas that are primitively assumed to have classes corresponding to them in NF. In ML, however, stratification is not necessary for the specification of ultimate classes but only for classes .that are elements, i.e., classes whose individuation is not in question (regardless of whether they are grounded or not). Thus, given Frege's definition of natural number, mathematical induction is provable in $\mathbf{M L}$ without the restriction to stratified formulas that is required in $\mathbf{N F}$.

The problem with this solution is that, unlike the situation in NF, where all classes are 'elements', or 'sets', we cannot prove that the class of natural numbers is a 'set', i.e., that it does not have a faulty individuation. We can, of course, simply assume as an additional axiom that the class of natural numbers is a 'set'; but, as has been argued by others, if we can add axioms to $\mathbf{M L}$ in this way, why not just stick with $\mathbf{N F}$ and add an axiom to the effect that mathematical induction holds for all formulas, stratified or otherwise? (Cp. Fränkel et al., 1973, p. 171.) Why assume that there are classes at all whose individuation is a problem? Or, more to the point, at least from Quine's perspective, why assume that there are objects (i.e., values of bound individual variables) that cannot be members of any class at all, not even of their singletons? No paradox is forthcoming on the assumption that there are at least finite classes of ultimate classes, e.g., singletons, doubletons, etc., though of course allowing that would mean giving up Quine's analysis of 'set'. What, then, is the real ontological point of that analysis?

Quine does not exclude the identity context in his talk of ultimate classes, it should be noted, for he maintains that the axiom of extensionality, which in $\mathbf{M L}$ has the same unqualified form as in NF, amounts to a principle of individuation for classes, ultimate or otherwise. In other words, ultimate classes have the same principle of individuation as the classes that are 'sets'. But, then, how are we to explain the difference between classes that are 'sets' and those that are not, i.e., those that are ultimate? Clearly, short of being merely a form of dogma, there has to be an explanation for the difference between ultimate classes and classes that are 'sets' - especially if ultimate classes are taken as a way of dealing with such twilight entities as concepts. Again, what is the real ontological import of Quine's analysis of 'set'? And, given his commitment even in ML to construing all objects as classes, what does that analysis have to say about the being of objects whose individuation is not in question?

There are good answers to these questions, but they cannot be found in 
ML itself. For, the problem here seems once again to be Quine's insistence on identifying predication with membership and concepts with classes, some of which are now said to be ultimate while others are not. Thus, in identifying predication with membership and concepts with classes, ultimate or otherwise, Quine in effect reduces all second-order notions (such as the functional role of concepts as unsaturated predicable entities and the sui generis referential role of predicate quantifiers regarding such entities) to first-order notions, thereby representing concepts as objects (i.e., as values of bound individual variables) and predicate quantifiers as first-order objectual quantifiers. The solution to Quine's problems with $\mathbf{M L}$ is not to identify predication with membership and concepts with classes, ultimate or otherwise, but to return to our old foundations and get rid of ultimate classes in favor of concepts. We need to return to a Fregean view of logic (or a variant thereof), in other words, where concepts are unsaturated predicable entities and membership is based on predication and not a substitute for it.

The simplest way to describe the kind of Fregean view we have in mind here is first to return to our old foundations of logic with nominalized predicates as abstract singular terms as represented by $\mathbf{H S} \mathbf{T}^{*}$, which, as already noted, is the framework that explained the oddities of Quine's new foundations, NF. Also, let us conservatively extend HST* by introducing $\lambda$ abstracts into it as complex predicate expressions. For obvious reasons, we call the result of extending $\mathrm{HST}^{*}$ in this way $\boldsymbol{\lambda H S T}^{*}$. Note that, like simple predicates (i.e., predicate variables and constants), all $\lambda$-abstracts have a pair of parentheses (and commas in the case of a relational predicate) surrounding their subject positions, and, as with simple predicates, they are nominalized and transformed into abstract singular terms by simply deleting their accompanying pair of parentheses (and commas). Also, as predicate expressions, all $\lambda$-abstracts are taken as legitimate substituends of the bound predicate variables, which, logistically, can be succinctly expressed as follows (where $F$ is not free in $\varphi$ ):

$$
(\exists F)\left(\left[\lambda x_{1}, \ldots x_{n} \varphi\right]=F\right) .
$$

This formula, which can itself be taken as a more fundamental form of comprehension principle, yields, on the basis of Leibniz's law, the more familiar comprehension principle,

$$
(\exists F)\left(\forall x_{1}\right) \ldots\left(\forall x_{n}\right)\left(F\left(x_{1}, \ldots, x_{n}\right) \leftrightarrow \varphi\right) .
$$

In HST $^{*}$, however, the latter must be homogeneously stratified, which means that in $\mathbf{A H S T}^{*}$, as a conservative extension of $\mathbf{H S T}^{*}$, all $\lambda$-abstracts, as 
a matter of logical grammar alone, must also be homogeneously stratified, since otherwise we would be able to prove a comprehension principle that goes beyond what we can prove in $\mathbf{H S T}^{*}$.

Now, from a conceptual point of view, the exclusion of inhomogeneouslystratified $\lambda$-abstracts from the class of grammatically well-formed predicate expressions seems inappropriate and counter-intuitive. How, in particular, could Russell even have formulated his paradox of predication if he could not form a concept corresponding to the complex predicate $[\lambda x(\exists G)(x=$ $G \wedge \neg G(x))$ ], which obviously is not homogeneously stratified ${ }^{13}$ Here, of course, we must keep in mind that, contrary to Russell's view of the matter, concepts are unsaturated predicable entities and not independently real objects. Thus, in particular, it is one thing to maintain that the Russellian predicate, $[\lambda x(\exists G)(x=G \wedge \neg G(x))]$, when nominalized and construed as an abstract singular term, fails to denote any object at all, and altogether quite another to maintain that the same predicate is meaningless in its role as a predicate and cannot stand for a concept. Unlike Quine's move from NF to ML, in other words, there are good reasons, independent of the issue of being able to prove an unqualified version of mathematical induction, to modify the logic of $\boldsymbol{\lambda H S T}^{*}$ by allowing all $\lambda$-abstracts to be well-formed predicate expressions.

The point of the modification of $\boldsymbol{\lambda} \mathbf{H S T}{ }^{*}$ that we are proposing here is that on a Fregean view of concepts (or a variant thereof, such as we will consider later) there is a difference between the laws of compositionality for conceptformation and the laws regarding the conditions under which concepts, as unsaturated predicable entities, have objects, e.g., extensions, corresponding to them. This means that the thesis that every singular term (including nominalized predicates) of our logical language must denote can no longer be taken as a law of logic; that is, we must replace the standard first-order logic that is part of $\boldsymbol{\lambda H S T}^{*}$ by a first-order logic that is free of existential presuppositions for singular terms. In this way, in regard to Russell's paradox of predication, we can maintain, on the basis of our more fundamental form of comprehension principle, that there is indeed a concept corresponding to the Russell predicate, i.e.,

$$
(\exists F)([\lambda x(\exists G)(x=G \wedge \neg G(x))]=F),
$$

\footnotetext{
${ }^{13}$ For this version of Russell's paradox, see Russell (1937, p. 97), where he describes the concept in question in terms of "what seems like a complex relation, namely the combination of nonpredicability with identity".
} 
even though that concept has no object corresponding to it as its conceptcorrelate (e.g., as its extension); that is, even though,

$$
\neg(\exists y)([\lambda x(\exists G)(x=G \wedge \neg G(x))]=y),
$$

is also provable (as a result of Russell's argument). Which concepts do have objects corresponding to them as their concept-correlates? Well, minimally, we can assume, analogous to Quine's extension of $\mathbf{N F}$ to $\mathbf{M L}$, that all of the objects denoted by nominalized predicates in $\boldsymbol{\lambda H S T}^{*}$ are also denoted by nominalized predicates of the new logic, which we call $\mathbf{H S T} \mathbf{\lambda}_{\lambda}^{*}$ Both $\boldsymbol{\lambda} \mathbf{H S T} \mathbf{S}^{*}$ and $\mathbf{H S T}_{\lambda}^{*}$, incidentally, as alternative reconstructions of our old foundations for logic as based on predication, can be shown to be consistent relative to weak Zermelo set theory. ${ }^{14}$

The questions we raised about Quine's system ML can now be answered in terms of the logical system $\mathbf{H S T} \mathbf{T}_{\lambda}^{*}$ or, rather, in terms of (monadic) $\mathbf{H S T}_{\lambda}^{*}+$ $\left(\mathbf{E x t}^{*}\right)+\left(\mathbf{Q}^{*}\right)$, where the objects corresponding to concepts are taken to be classes in the logical sense, i.e., classes as the extensions of those concepts. We include Quine's thesis, $\left(\mathbf{Q}^{*}\right)$, that all objects are classes not because we think that it is true (we don't) but because that is what Quine assumes in adopting his unqualified axiom of extensionality, (ext), even in ML. Now, from the perspective of this new version of our old foundations for logic, all of the classes of $\mathbf{M L}$, ultimate or otherwise, are really concepts misconstrued as objects. Many, even if not all, of the concepts of $\mathbf{H S T}_{\lambda}^{*}$ have objects corresponding to them, which, on the assumption of the extensionality axiom $\left(\mathbf{E x t}^{*}\right)$, can be taken as the extensions of those concepts. Those extensions, or classes, turn out to be the objects referred to by Quine as 'sets', because in fact it is true of all objects whatsoever in $\mathbf{H S T}_{\lambda}^{*}+\left(\mathbf{E x t}^{*}\right)$ that they belong to some class or other, which on our Fregean analysis of membership means only that they fall under some concept or other. But in $\mathbf{H S T}_{\lambda}^{*}$, as an alternative reconstruction of our old foundation for logic, and in particular as a reconstruction that is free of existential presuppositions regarding singular terms, to fall under some concept or other is the same as to be an object, i.e., to be the value of a bound individual variable:

$$
\vdash_{\mathbf{H S T}_{\lambda}^{*}}(\exists x)(a=x) \leftrightarrow(\exists F) F(a),
$$

\footnotetext{
${ }^{14}$ Cf. Cocchiarella (1987, chap. 2, sec. 14, or chap. 4, sec. 7) for a detailed formal description of the system $\mathbf{H S T}_{\lambda}^{*}$ and for a proof of its consistency relative to weak Zermelo set theory.
} 
where $a$ is a singular term in which $x$ is not free. ${ }^{15}$ In other words, the real ontological import of Quine's definition of 'set' is simply an alternative analysis of what it is to be an object simpliciter.

Ultimate classes, on this interpretation, are not objects of all, but are really those concepts, such as the one represented by the Russell predicate, $[\lambda x(\exists G)(x=G \wedge \neg G(x))]$, that have no objects corresponding to them as their extensions. It is because ultimate classes are not objects to begin with that they cannot be members of other classes, not even of their singletons, since to be a member of a singleton is to fall under a concept and therefore be an object after all. Of course, not being objects, ultimate classes have the same individuation problem that concepts have as unsaturated predicable entities, which is to say no problem at all, because the question of their 'individuation' can be taken seriously only by misconstruing them as objects.

This does not mean that Quine's dictum, "no entity without identity", must be rejected; rather, it means only that the notion of identity involved in that dictum must not be restricted to 'individuals', i.e., to individuated entities, or, in modern parlance, to objects as values of bound individual variables. Thus, if identity is construed as a transcendental notion in the scholastic sense, as in fact it is construed in $\mathbf{H S T}_{\lambda}^{*}$ as an alternative reconstruction of our old foundations for logic, then even concepts, and therefore ultimate classes as well on this interpretation, can have their identity despite

\footnotetext{
${ }^{15}$ It should be noted that in $\mathbf{H S T}_{\lambda}^{*} \neg(\exists y)(a=y)$ is not provably equivalent to $[\lambda x \neg(\exists y)(x=y)](a)$, because the latter formula, if true (per impossibile), involves being the value of an individual variable (viz., $x$ ) bound by the $\lambda$-operator, i.e., it involves being the value of a bound individual variable after all.
} 
not being well-individuated entities, that is, despite not being objects. ${ }^{16}$

The reduction of $\mathbf{M L}$ to (monadic) $\mathbf{H S T} \mathbf{T}_{\lambda}^{*}+\left(\mathbf{E x t} \mathbf{t}^{*}\right)+\left(\mathbf{Q}^{*}\right)$ can be described formally in terms of a simple translation function, $\operatorname{tr} s$, that replaces all of the individual variables occurring in the formulas of $\mathbf{M L}$ by one-place predicate variables (where distinct individual variables are replaced by distinct predicate variables). Every formula of $\mathbf{M L}$, in other words, becomes a formula of monadic $\mathbf{H S T}_{\lambda}^{*}$ in which no individual variable occurs at all, which indicates our point that $\mathbf{M L}$ is only about (monadic) concepts and the extensions (if any) that are their correlates. It is now easily shown that if $\varphi$ is a theorem of $\mathbf{M L}$, then the translation of $46, \operatorname{tr} s(\varphi)$, is a theorem of $\mathbf{H S T}_{\lambda}^{*}+\left(\mathbf{E x t}^{*}\right)+\left(\mathbf{Q}^{*}\right)$. (Cf. Cocchiarella (1985b, sec. 8) for the details of a proof of this claim.)

There is no need, of course, to retain Quine's thesis, $\left(\mathbf{Q}^{*}\right)$, that all objects are classes (and 'sets' in particular) in the framework of $\mathbf{H S T}_{\lambda}^{*}$, where its status as a false metaphysical thesis is rendered perspicuous. In dropping $\left(\mathbf{Q}^{*}\right)$ we are no longer able to prove Quine's unqualified version of the extensionality axiom, (ext), but that is really as it should be. After all, if extensionality really is an assumption that we want to make in this reconstruction of our old foundations for logic, then the natural form of that assumption is our second-order version of the extensionality axiom, (Ext*). In any case, given that assumption, the objects denoted by nominalized predicates, as already noted, are none other than the extensions of the concepts those predicates otherwise stand for in their role as predicates. Not all concepts will have

\footnotetext{
${ }^{16}$ Allowing a transcendental notion of identity marks a departure from Frege's view, we agree. We can avoid such a departure, if we wish, by simply dropping identity in favor of indiscernibility, as is done in the version of $\mathbf{H S T}_{\lambda}^{*}$ formulated in the first part of Cocchiarella (1985a).

With identity as a transcendental notion, it is noteworthy that although it is provable that objects that are indiscernible are therefore identical, i.e.,

$$
\vdash_{\mathbf{H S T}_{\lambda}^{*}}(\forall x)(\forall y)(x \equiv y \leftrightarrow x=y),
$$

where ' $\equiv$ ' is defined as indiscernibility), the same cannot be said of concepts. In fact, just the opposite holds, that is,

$$
\vdash_{\mathbf{H S T}_{\lambda}^{*}}(\exists F)(\exists G)(F \equiv G \wedge F \neq G) .
$$

In particular, concepts that have no objects (e.g., extensions) corresponding to them as their concept-correlates, vacuously fall under all of the same concepts and, therefore, are indiscernible, even though they are not the 'same' concepts. For more on this, see Section 9 of the paper cited above.
} 
an extension corresponding to them, to be sure, which means that, in this respect at least, Frege did overestimate the kinship between predication and membership. That is, the second-order comprehension principle for membership,

$$
(\forall F)(\exists y)(\forall x)(x \in y \leftrightarrow F(x)),
$$

is refuted in $\mathbf{H S T}_{\lambda}^{*}+\left(\mathbf{E x t} \mathbf{t}^{*}\right.$ ) (or even in just $\mathbf{H S T} \mathbf{T}_{\lambda}^{*}$ ), given our Fregean analysis of membership. The laws of compositionality for concept-formation, in other words, cannot in all cases be transformed into laws regarding the conditions under which concepts, as unsaturated predicable entities, have extensions corresponding to them. Frege was right in maintaining that it is by means of our logical faculties that we can "lay hold upon the extension of a concept, by starting out from the concept" (Frege 1979, p. 181), but he was wrong to think that every concept will have an extension.

In addition to those concepts for which it can be proved that they have no extension corresponding to them, as well as those for which it is provable that such an extension does exist, there are many concepts in the framework of $\mathbf{H S T}_{\lambda}^{*}+\left(\mathbf{E x t}^{*}\right)$ for which it is neither provable nor disprovable that those concepts have a corresponding extension. In particular, it is neither provable nor disprovable that the Fregean concept of a natural number, i.e., the concept represented by the following $\lambda$-abstract (where $S$ stands for the successor relation),

$$
[\lambda x(\forall F)(F(0) \wedge(\forall y)(\forall z)[F(y) \wedge S(y, z) \rightarrow F(z)] \rightarrow F(x))]
$$

has an extension corresponding to it as its concept-correlate. That is, where $N$ is now defined as abbreviating this $\lambda$-abstract, the following existence claim, is neither provable nor disprovable in $\mathbf{H S T}_{\lambda}^{*}+\mathbf{E x t}^{*}$ ) (with, or without, $\left.\left(\mathbf{Q}^{*}\right)\right) .{ }^{17}$ While this is a plausible existence claim to make, nevertheless, adding it to $\mathbf{H S T}_{\lambda}^{*}+\left(\mathbf{E x t}^{*}\right)$ as a postulate does mean going beyond the kinds of existence claims that can be validated on the basis of the laws of logic as represented by this system. Indeed, this seems to be the point where mathematics begins to transcend logic in just the kinds of existential posits that it makes. Note, however, that this does not mean that mathematical concepts are nonlogical concepts about the physical world, that is, that mathematics involves applying logic by adding descriptive constants to it that are not analyzable in purely logical terms. Rather, it means only that mathematics

\footnotetext{
${ }^{17}$ This claim is based on a proof of the related claim for ML in Rosser (1952).
} 
transcends logic by assuming the existence of extensions (or even of intensions in the case of intensional mathematics) as the objects corresponding to certain concepts that otherwise cannot be proved on logical grounds alone to have a concept-correlate. Logicism is true, in other words, only in the sense that all of the concepts of classical mathematics can be given a purely logical analysis, but it is false in that not all of the posits of classical mathematics can be validated on purely logical grounds alone. It is in this way that we see where logic leaves off and mathematics begins, in other words, and not in Quine's way of taking logic to consist only of elementary, first-order logic and mathematics as a first-order 'set' theory, with or without ultimate classes.

\section{Frege Versus Quine on Higher-Order Logic}

According to Quine, the framework of higher-order logic

"has the fault . . . of diverting attention from the major cleavages between logic and set theory. It encourages us to see the general theory of classes and relations as a mere prolongation of quantification theory in which the hitherto schematic predicate letters are newly admitted into quantifiers and into other positions that we hitherto reserved to ' $x$ ', ' $y$ ', etc." (Quine 1963, p. 257)

Here, by the "other positions" that are reserved for the individual variables, Quine means the subject positions that predicates bring with them in their role as predicates. Thus, his claim is that (1) by allowing quantifiers to be affixed to predicate variables we are committed to (2) allowing predicate variables, and predicate expressions in general, to be nominalized and occur as abstract singular terms on a par with individual variables and, furthermore, (3) that the objects (viz., classes) denoted by such abstract singular terms are the same entities that are the values of the bound predicate variables. A secondary claim, implicit in Quine's use of 'hitherto', seems to be (4) that the use of predicate variables as dummy schema letters in first-order logic antedates their use in the predicate quantifiers of higher-order logic.

In regard first to the secondary claim (4), let us note that by Quine's lights, our entire line of thought about predication and the logical notion of a class "springs from a wrong philosophy," and "the orthodox terms "first-order predicate logic' and 'higher-order predicate logic' have the same deplorable 
source," namely, "a blurred view of ... the distinction between schematic letters and variables, and ... between use and mention" (Hahn and Schilpp 1986, 591ff.). Anyone who quantifies with respect to predicate variables, Quine insists, fails to see, or sees only "dimly," that he is really assuming "new objects as values of the newly quantified variables" (ibid., p. 592; emphasis added). Quantifying with respect to predicate variables, in other words, at least where those variables are assumed to have values, amounts to a confusion of substitutional quantification with objectual quantification.

What Quine says here about a confusion between schematic letters and variables, and between mention and use, is not only false but misleading as well. It is false, in particular, that the use in first-order logic of predicate variables as dummy schema letters antedates the use in higher-order logic of quantifiers binding those variables. It is false because Frege, the founding father of first-order quantificational logic, gave us that theory only as an integral part of his second-order logic (i.e., the logic of his Begriffsschrift). It is also misleading, because, by inverting the original order of the development of mathematical logic, it tries to present the development of higher-order logic as a mistake based on a confusion between schematic letters and variables, and between mention and use. Bringing in mention and use confusions is an important tactic of Quine's, but, as in his application of that tactic in modal logic, it simply begs the question at issue and amounts to an ad hominem form of argument. For it is only by seeing things 'dimly' and having a 'blurred view' of important distinctions, according to Quine, that one can adopt the positions he decries.

In regard to Quine's more important claim, namely, (1) that we cannot affix quantifiers to predicate variables unless (2) those variables are nominalized and allowed to occur as abstract singular terms, and (3) that the objects denoted by such abstract singular terms must be the values of the bound predicate variables, let us note that Frege did not admit abstract singular terms at all into his original formulation of second-order logic in his Begriffsschrift. It was only later, in the Grundgesetze, that such terms could be generated through the use of the smooth-breathing abstraction operator as a nominalizing device. That was an important and novel step to take, we agree, and, as Frege observed, in order "to obtain objects out of concepts, namely, extents of concepts or classes" (Frege 1910, p. 361), it was a necessary step as well. Nevertheless, Frege was clear and explicit on this being an added step, for, he insists that "we can treat the principal parts of logic without speaking of classes, as I do in my Begriffsschrift" (ibid., p. 360). The 
logic of the Begriffsschrift includes predicate, as well as first-order, quantifiers, and although predicates are taken to stand for concepts and relations, they are emphatically not to be taken as names or abstract singular terms (i.e., as saturated expressions). Thus, from a Fregean point of view, Quine is wrong in claiming that we cannot affix quantifiers to predicate variables unless those variables are also allowed to occur as abstract singular terms. $\mathrm{He}$ is also wrong on this interpretation in claiming that the objects denoted by nominalized predicates as abstract singular terms must be the same entities as are taken to be the values of the bound predicate variables. For the objects (viz., classes) denoted by such abstract singular terms in Frege's Grundgesetze are not the unsaturated concepts and relations that are taken as the values of the bound predicate variables.

It is noteworthy that in commenting on Frege's logic, Quine erroneously claimed that Frege "construed a general term as naming its extension" (Quine 1951, p. 90), where "to say that Frege treats general terms as names of classes ... is simply to say that he provides for substitution of general terms for quantified class variables" (ibid., p. 93). Predicate quantifiers are a "defect" in a theory of reference such as Frege's, Quine maintained, and he also claimed that it is he who has clarified matters by keeping "general terms distinct from abstract singular terms" (ibid., p. 94). "In depriving general terms or predicates of the virtues of names," Quine wrote, apparently completely unaware that he is now voicing Frege's own view of the matter, "I do not thereby deny that there are certain classes connected with them otherwise than in the fashion of being named" (ibid., p. 95).

These views about predicate quantifiers and the distinction between predicates and their nominalizations as abstract singular terms also occur in Quine's 1947 paper, 'On Universals', but there they are compounded with an argument, namely, Russell's paradox of predication, that completely fails to hit its mark and that in fact is fallacious on Quine's formulation. Thus, beginning with the observation (which is not here in dispute) that "if we bind the schematic predicate-letters of quantification theory," that is, of first-order predicate logic (without identity), "we achieve a reification of universals," Quine goes on to claim that "these universals are entities whereof predicates may thenceforward be regarded as names" (Quine 1947, p. 77). Now it is precisely this claim that we have challenged, for, as in Frege's Begriffsschrift, predicates do not become names just because they stand for values of bound predicate variables. Predicates can be nominalized, we agree, i.e., they can be transformed into abstract singular terms in just the way described in Sec- 
tion 2 above, and this in fact is just what Quine proposes "in order to take in the theory of classes" (ibid., p. 78). That is, we are to "allow the predicate variables all privileges of the [individual] variables ' $x$ ', ' $y$ ', etc. The predicate letters, when thus admitted to quantifiers, acquire the status of variables taking classes as values" (ibid.). Quine then makes his by now familiar identification of predication with membership: "the notation ' $F x$ ' (or ' $F G$ ', etc.), when ' $F$ ' is bound, comes to mean that $x$ (or $G$, etc.) is a member of the class $F "$ (ibid.). ${ }^{18}$

Note that the logic now being described is essentially the logical system HST $^{*}$ described in Section 2, but without the homogeneously stratified comprehension principle, (HSCP*), as an added axiom schema. This logic, or proper subsystem of $\mathbf{H S T}^{*}$ (without identity), is a variation of a system called $\mathbf{T}_{0}^{*}$ (in Cocchiarella 1973), and $\mathbf{T}_{0}^{*}$, as is shown there, can be given a trivial consistency proof relative to the extended propositional calculus, which in turn is reducible to propositional logic simpliciter. ${ }^{19}$ In other words, $\mathbf{T}_{0}^{*}$ can be shown to be absolutely consistent and not just relatively consistent. How strange it is then to read Quine's claim that Russell's paradox of predication can be derived in this logic (op. cit., p. 78) and that it is therefore inconsistent, thereby showing, supposedly, the impropriety of quantifying and nominalizing the role of predicates.

Quine's argument, needless to say, is fallacious. The critical step is his inference from

$$
(\exists F)(\forall H)(F(H) \leftrightarrow G(H)),
$$

which is provable in $\mathbf{T}_{0}^{*}$, the logic in question, to

$$
(\exists F)(\forall H)(F(H) \leftrightarrow \neg H(H)),
$$

\footnotetext{
${ }^{18}$ Quine does not use parentheses, as we do, as integral parts of predicates to perspicuously mark off the subject positions that predicates bring with them in their role as predicates. Still, the functional role of predicates is indicated by their positions in basic or atomic formulas. On the other hand, the claim that predicates are names and that predication is membership does suggest that Quine may be confusing predicate positions in atomic formulas with subject positions.

${ }^{19}$ The system $\mathbf{T}_{0}^{*}$ is described in Cocchiarella (1973, Sec. 2). Its consistency proof amounts to simply deleting the subject positions (and the singular terms oc positions) of each predicate occurring in a formula, thereby translating each predicate variable in a predicate position into a propositional variable in a propositional position, resulting ín a formula of the extended propositional calculus. It ís then easily seen that a formula is provable in $\mathbf{T}_{0}^{*}$ only if its transform is provable in the extended propositional calculus.
} 
which is not provable. The fallacy in question involves substituting an open formula, e.g., $\neg H(x)$, in which $H$ has a free predicate position occurrence, for the free occurrence of $G$ in (A) that occurs within the scope of the predicate quantifier $(\forall H)$, thereby replacing the free predicate position occurrence of $G$ by a bound predicate position occurrence of $H .^{20}$

The claim, which Quine does not himself make, that (B) is really an instance of a comprehension principle is also erroneous, because no comprehension principle at all was assumed for this framework. Adding the homogeneously-stratified comprehension principle, $\left(\mathbf{H} \mathbf{S C P} \mathbf{C P}^{*}\right)$, to $\mathbf{T}_{0}^{*}$, as already noted, results in the system $\mathbf{H S T}^{*}$, which is consistent relative to weak Zermelo set theory. But, then, (B) is clearly not an instance of ( $\left.\mathbf{H S C P}^{*}\right)$; nor, for that matter, is it even an instance of a heterogeneously-stratified comprehension principle. More importantly, (B) is not even an instance of the unstratified comprehension principle,

$$
\left(\exists F^{n}\right)\left(\forall x_{1}\right) \ldots\left(\forall x_{n}\right)\left(F\left(x_{1}, \ldots, x_{n}\right) \leftrightarrow \varphi\right),
$$

which (where $F^{n}$ is not free in $\varphi$ ) is the comprehension principle of standard second-order logic (without identity) extended so as to allow nominalized predicates to occur as abstract singular terms in the formulas in question. In particular, (B) should not be seen as derived from an instance of $\left(\mathbf{C P}^{* *}\right)$ of the form,

$$
(\exists F)(\forall x)(F(x) \leftrightarrow \neg x(x)),
$$

because the latter, which contains an individual variable in a predicate position, is not even grammatically well-formed. Adding $\left(\mathbf{C P}^{* *}\right)$ to $\mathbf{T}_{0}^{*}$, the logical theory in question, results in the logic called $\mathbf{T}^{*}$ (without identity) (in Cocchiarella 1973), and $\mathbf{T}^{*}$, as is shown there, is a conservative extension of standard second-order logic, which means that $\mathbf{T}^{*}$ also has a trivial consistency proof. ${ }^{21}$

It also cannot be claimed in this context, incidentally, that (B) is an existential generalization of a definition of what it is for a concept to be 'impredicable', i.e., a definition of the form:

$$
\operatorname{Impred}(H) \leftrightarrow \neg H(H) .
$$

\footnotetext{
${ }^{20}$ For a detailed analysis of this fallacy and of the notion of the proper substitution of a formula for a predicate variable that is involved here when nominalized predicates are allowed to occur as abstract singular terms, see Cocchiarella (1973, sec. 3).

${ }^{21}$ Cf. Cocchiarella (1973, Sec. 6) for a proof of the claim that $\mathbf{T}^{*}$ (with or without an axiom of infinity) is a conservative extension of standard second-order predicate logic (with or without the same axiom of infinity).
} 
What is wrong with this claim is that a definition must be noncreative, which means that if a contradiction is provable on the basis of a definitional extension of a theory, then a contradiction must already be provable in that theory independently of any use of the definition in question. But whether or not the logical background contains a comprehension principle, stratified or otherwise, that is, whether it be $\mathbf{T}_{0}^{*}, \mathbf{T}^{*}$, or $\mathbf{H S T} \mathbf{T}^{*}$, it is in all of these cases known to be consistent, and therefore the so-called definition in question is not really a definition at all, but merely a spurious assumption that leads to a contradiction. ${ }^{22}$

Finally, it should also be noted that $(\mathbf{B})$ cannot be derived in $\mathbf{T}^{*}$ from

$$
(\exists F)(\forall x)(F(x) \leftrightarrow(\exists G)[x=G \wedge \neg G(x)]),
$$

because identity has not been taken as a primitive notion of this system. There is, of course, indiscernibility (as represented by ' $\equiv$ '), which is definable in $\mathbf{T}^{*}$ in the usual way and, hence, there is the following,

$$
(\exists F)(\forall x)(F(x) \leftrightarrow(\exists G)[x \equiv G \wedge \neg G(x)]),
$$

as an instance of the comprehension principle (CP $\left.{ }^{* *}\right)$. Applying Russell's argument in this case does not lead to a contradiction, however, but only shows that in $\mathbf{T}^{*}$ there are indiscernible concepts that are not coextensive, i.e.,

$$
\vdash_{\mathbf{T}^{*}}(\exists F)(\exists G)(F \equiv G \wedge \neg(\forall x)[F(x) \leftrightarrow G(x)]) .
$$

This may seem to be an odd, or counter-intuitive, result, but it must be remembered that indiscernibility is a relation between objects (concept-correlates in this case) and that, unlike identity, it cannot be a transcendental notion covering concepts as well. Frege, we agree, rejected the idea of identity as a transcendental notion different from indiscernibility and, of course, he would have rejected any framework in which non-coextensive concepts can have indiscernible objects as their correlates. That does not mean, however, that

\footnotetext{
${ }^{22}$ One thing that is wrong with the 'definition' of Impred is that it gives no truth conditions for objects other than concept-correlates; that is, it is not defined for all objects whatsoever, but only for those that are the correlates of concepts. The whole point of a second-order predicate logic with nomínalízed predicates as abstract singular terms is that predicates can be meaningfully applied to all singular terms, regardless whether the latter denote concrete or abstract objects, and regardless whether those predicates are logically false of those objects or not.
} 
there cannot be a modified Fregean framework in which the objects correlated with concepts are not their extensions but, for example, their cardinal numbers instead, in which case there would be nothing really odd about there being non-coextensive concepts that have indiscernible objects (i.e., the 'same' cardinal number) as their correlates. In any case, the important point is that what is involved here is indiscernibility (or 'identity' between objects) and not identity as a transcendental notion (i.e., not identity between concepts).

We can add identity as a transcendental notion to the framework of $\mathbf{T}^{*}$, but, then, on pain of contradiction otherwise, we must not allow it to occur in instances of the comprehension principle $\left(\mathbf{C P}^{* *}\right)$. Similarly, in adding $\lambda$ abstracts to the logical grammar of $\mathbf{T}^{*}$, to obtain the system $\boldsymbol{\lambda} \mathbf{T}^{*}$. the way that $\boldsymbol{\lambda} \mathbf{H} \mathbf{S T}^{*}$ was obtained from $\mathbf{H S T}^{*}$, we must exclude the identity sign from occurring in those $\lambda$-abstracts. It will then be provable in $\boldsymbol{\lambda} \mathbf{T}^{*}$ (with identity) that identity does not stand for a relation between objects the way that indiscernibility does, and, by the above result about indiscernibility, that there are distinct (nonidentical) concepts that have indiscernible conceptcorrelates:

$$
\vdash_{\boldsymbol{\lambda} \mathbf{T}^{*}}(\exists F)(\exists G)(F \equiv G \wedge F \neq G) .
$$

Russell's paradox of predication, as these observations indicate, seems ineluctably to involve identity and the complex concept represented by the $\lambda$-abstract, $[\lambda x(\exists G)(x=G \wedge \neg G(x))]$. This abstract, as already noted, is not grammatically well-formed in either $\boldsymbol{\lambda} \mathbf{T}^{*}$ (with identity) or $\boldsymbol{\lambda} \mathbf{H S T} \mathbf{T}^{*}$ (the logic that contains a natural interpretation of Quine's 'set' theory $\mathbf{N F}$ ), and although it is grammatically well-formed in $\mathbf{H S T}_{\lambda}^{*}$ (the logic that contains a natural interpretation of Quine's 'set' theory $\mathbf{M L}$ ), where it stands for a concept (as an unsaturated predicable entity), nevertheless, upon nominalization, it fails to denote any object as the correlate of that concept. Similarly, we can modify the first-order logic part of $\boldsymbol{\lambda} \mathbf{T}^{*}$, so as to render it free of existential presuppositions regarding singular terms, the way we modified $\boldsymbol{\lambda} \mathbf{H S T} \mathbf{T}^{*}$ to obtain $\mathbf{H S T} \mathbf{T}_{\lambda}^{*}$. In the resulting system, $\mathbf{T}_{\lambda}^{*}$, the Russell predicate now stands for a concept, just as it does in $\mathbf{H S T}_{\lambda}^{*}$, but as in $\mathbf{H S T}_{\lambda}^{*}$ that same predicate, when nominalized, simply fails to denote. ${ }^{23}$ These different

\footnotetext{
${ }^{23}$ Cf. Cocchiarella (1986a, chap. 4, sec. 6) for a consistency proof of $\mathbf{T}^{*}$ with identity. The formal description and consistency proofs for $\boldsymbol{\lambda} \mathbf{T}^{*}$ and $\mathbf{T}_{\lambda}^{*}$ (with identíty) as well as of $\boldsymbol{\lambda} \mathbf{H S T} \mathbf{T}^{*}$ and $\mathbf{H} \mathbf{S} \mathbf{T}_{\lambda}^{*}$, are given in chapters 5 and 6 , together with a Frege for all of these different systems.
} 
systems constitute the different options that are available as a response to Russell's paradox of predication, assuming, that is, that we want to allow for an impredicative comprehension principle covering the kinds of complex concepts involved in Russell's argument in the first place.

Quine himself, it should be noted, avoids taking identity as a primitive logical concept and in general attempts to explain it in terms of other notions instead. In NF, for example, Quine defines identity as membership in all the same classes, which on our analysis amounts to indiscernibility, i.e., as falling under all of the same concepts. That definition suffices in NF, as it does in HST* (without identity), for many of the purposes of identity, but it may be questioned whether it really amounts to identity. With identity as primitive, for example, the following,

$$
F(x) \leftrightarrow(\exists z)[x=z \wedge F(z)],
$$

is a valid formula and, therefore, were identity taken as a primitive logical concept of $\mathbf{N F}$, the stratified comprehension principle of $\mathbf{N F}$ would be equivalent to

$$
(\exists y)(\forall x)(x \in y \leftrightarrow(\exists z)[x=z \wedge F(z)]),
$$

where $F$ has only stratified formulas (in which neither $y$ nor $x$ have free occurrences) as its substituends. With identity defined as membership in all the same classes, however, that is, as 'indiscernibility' (for which we will continue to use ' $\equiv$ '), replacing the stratified comprehension principle of $\mathbf{N F}$ by

$$
(\exists y)(\forall x)(x \in y \leftrightarrow(\exists z)[x \equiv z \wedge F(z)]),
$$

where the substituends of $F$ are restricted as above, results in a system that is decidedly weaker than $\mathbf{N F}$, and in fact it results in a system that can be proved consistent relative to weak Zermelo set theory (cf. Cocchiarella 1976). In this weaker system, moreover, membership in all the same classes, i.e., indiscernibility, does not imply co-extensivity, and in fact the claim that there can be indiscernible classes that are not coextensive, i.e.,

$$
(\exists x)(\exists y)(x \equiv y \wedge \neg(\forall z)[z \in x \leftrightarrow z \in y]),
$$

can be true in models of this weaker system. This is perhaps not so surprising when we notice that every instance of the weaker comprehension principle is derivable in $\mathbf{T}^{*}$, where it is provable that there are non-coextensive concepts that have indiscernible objects as their correlates. Of course, what makes 
this result so odd in the weakened version of $\mathbf{N F}$ is that the unqualified extensionality axiom is also assumed there. Clearly, these results should not be possible if Quine's definition represented an adequate analysis of identity.

\section{Conceptualism Versus Nominalism as Formal Theories of Predication}

There is an interpretation of predicate quantifiers under which predicates are not construed as names and predicate variables are not taken as having classes as their values, which, in recent years, Quine seems willing to allow. This is the so-called substitutional interpretation, according to which bound predicate variables have no values at all but only substituends, namely, open formulas that do not themselves contain any bound predicate variables. Firstorder quantifiers, which are still interpreted objectually, are in no way affected by such an interpretation, it should be emphasized, and, as Quine has pointed out, switching to a substitutional interpretation of first-order quantifiers as well is pointless because such an interpretation only shifts the problem of reference to singular terms, e.g., individual constants and definite descriptions. An objectual interpretation of first-order quantifiers not only makes clear our ontological commitments, but, in terms of those commitments, it also gives a logically perspicuous representation of the referential role of singular terms. It is when predicates are not themselves (mis)construed as singular terms, according to Quine, that predicate quantifiers can have no referential significance and, therefore, unless predicate quantifiers are banned entirely, the only interpretation that can be given them is substitutional. Thus, whereas a substitutional interpretation of first-order quantifiers only obfuscates the nature of reference, a substitutional interpretation of predicate quantifiers, in a context in which predicates are not themselves (mis)construed as singular terms, will have no bearing at all on the notion of reference. In addition, and perhaps more to the point of the tenor of Quine's philosophy, whereas a substitutional interpretation of first-order quantifiers will only nullify a nominalistic theory of predication (according to which predicates are the only 'universals' that can be predicated of objects), a substitutional interpretation of predicate quantifiers is acceptable precisely because it represents just such a nominalistic theory (cf. Cocchiarella 1986a, chap. 1).

Nominalistic scruples aside, however, too much should not be made of 
a substitutional interpretation of predicate quantifiers. For, as the basis of a second-order logic, such an interpretation really does not result in any increase of logical powers over first-order logic (cp. Quine 1970, p. 94). In particular, one important drawback, as Quine himself has pointed out, is that it precludes impredicative definitions (cp. Hahn and Schilpp 1986, p. 593). Formally, this means that the comprehension principle must be restricted so that no formula containing a bound predicate variable is allowed to 'comprehend' a predicative expression, i.e., to be itself construed as a predicative context. Thus, the restricted comprehension principle validated by a substitutional interpretation of predicate quantifiers amounts to taking as an axiom every instance of the following schema:

$$
\left(\exists F^{n}\right)\left(\forall x_{1}\right) \ldots\left(\forall x_{n}\right)\left[F\left(x_{1}, \ldots, x_{n}\right) \leftrightarrow \varphi\right],
$$

where $\varphi$ is a formula in which no predicate variable has a bound occurrence (and in which $F^{n}$ does not occur free and $x_{1}, \ldots, x_{n}$ are pairwise distinct individual variables). The appearance here of an existential posit regarding the existence of a universal in the quantifier prefix, $\left(\exists F^{n}\right)$, is just that, under a substitutional interpretation, namely, an appearance and nothing more. In an applied theory of the second-order logic in question, this principle, under such an interpretation, involves no ontological commitments beyond what one is already committed to in the use of the first-order formulas of that theory. That is, by interpreting predicate quantifiers substitutionally, (CP!) will not commit us ontologically to anything we are not already committed to in our use of first-order formulas, which is where the nominalistic scruples about the nature of predication come in. The second-order logic that is complete with respect to such a nominalistic interpretation of predicate quantifiers, as is shown elsewhere, is standard predicative second-order logic (cf. Cocchiarella 1980). ${ }^{24}$ Given its exclusion of impredicative definitions, the real question, aside from nominalistic scruples, is why we should adopt such a logic at all.

There is a referential interpretation of predicate quantifiers under which predicates are not to be confused with names and that also results in a predicative logic, that is, a logic that has a similar constraint imposed on its comprehension principle. But, unlike the situation with a substitutional interpretation of predicate quantifiers, impredicative definitions are not disallowed in this logic, even though they fail to specify a value of the bound

\footnotetext{
${ }^{24} \mathrm{An}$ informal sketch and defense of a substitutional interpretation of predicate quantifiers can be found in Parsons (1971).
} 
predicate variables. The framework for this interpretation is what has elsewhere been called constructive conceptualism, where the constructive aspect means only that the concepts in question cannot be specified impredicatively or, in other words, that 'predicative' concepts can be formed only in accordance with the so-called vicious circle principle (cf. Cocchiarella 1986a, chap. 2, secs. 2-5). By a (predicable) concept we mean here a cognitive capacity that is exercised in judgments, whether expressed overtly in the form of speech acts or not, and in particular a capacity that underlies our ability to follow a rule of language regarding the use of a predicative expression. It is by means of (predicable) concepts as cognitive capacities in this sense that we are able to characterize and relate objects in various ways in our use of language as a representational system; for as capacities underlying our use of predicate expressions, concepts determine the truth conditions that we associate with those expressions. Indeed, in general, our 'knowledge' of the rules of language is really a matter of our having concepts in the sense of cognitive capacities - namely, the capacities for knowing how to use the expressions governed by those rules - and our following those rules is really a matter of our exercising those concepts as capacities. Note, however, that having a concept in this sense, although it is a form of 'knowledge', is not a form of propositional knowledge. In particular, knowing how to follow a rule (in the sense intended here) does not mean knowing how to formulate the rule propositionally. Indeed, in general, in thought and in speech we know how to follow the rules of language without also knowing how to formulate them propositionally. Instead of being propositional, this kind of 'knowledge' is really a form of identity, because the rules for knowing how to use the expressions of a language, when construed as determinants of our linguistic behavior, are really the same as the cognitive capacities we exercise in following those rules, and having such a cognitive capacity in this sense is what we mean by having a concept. Thus, in particular, having a "predicative' concept means knowing how to follow the rule governing the use of a predicative expression.

As cognitive capacities that can be exercised by the same person at different times, as well as by different persons at the same time, concepts are not images or ideas in the sense of particular mental occurrences - nor are they (saturated) objects of any kind for that matter. As cognitive capacities that might in fact never be exercised at all, concepts are not objects or things and, in that regard, they cannot be taken as values of the bound individual variables. Rather, adopting Frege's terminology, though not his metaphysics, 
concepts are really unsaturated cognitive structures, which become saturated only when they are exercised in thought or speech and result in a mental act, which, when it is overtly expressed, is the same as a speech act. It is the exercise (or saturation) of a predicable concept in particular which is what informs such a mental act with a predicative nature.

We reject Quine's description of concepts as images or ideas, accordingly, as well as his claim that concepts belong to a "misty realm" for which it is better that we adopt "the nominalist strategy" and talk only the of words and language instead (Quine 1974, pp. 34ff.). In conceptualism, constructive or otherwise, predicate quantifiers are not interpreted nominalistically, i.e., substitutionally, but referentially in that bound predicate variables are taken as having concepts as unsaturated cognitive structures as their values. This is not the same notion of reference that is given to first-order (objectual) quantifiers, it must be emphasized but, rather, like Frege's notion of Bedeutung (or Andeutung) as applied to predicates (or predicate variables) as unsaturated expressions, it must be viewed as a mode of reference that is sui generis. In this regard, we also reject Quine's claim that predicate quantifiers, in a context in which predicates are not themselves misconstrued as abstract singular terms, can have no referential significance and can therefore only be interpreted substitutionally.

Despite the similarity with Frege's notion of reference for predicate quantifiers, concepts in constructive conceptualism, unlike the situation in Frege's logic, cannot be specified impredicatively but, rather, are constrained by laws of compositionality characteristic of a predicative logic, i.e., laws that are in accordance with the vicious circle principle. This does not mean that the comprehension principle regarding 'predicative' concepts is the nominalistic principle (CP!) and, in fact, unlike the situation in nominalism, which is committed to a logic of predicate quantifiers that is not free of existential presuppositions regarding predicate letters, predicate quantifiers in constructive conceptualism are free of just such presuppositions. For this reason, the comprehension principle that is validated in constructive conceptualism is even more restrictive than $(\mathbf{C P}$ !) and has the following form instead:

$$
\left(\forall G_{1}\right) \ldots\left(\forall G_{k}\right)\left(\exists F^{n}\right)\left(\forall x_{1}\right) \ldots\left(\forall x_{n}\right)\left(F\left(x_{1}, \ldots, x_{n}\right) \leftrightarrow \varphi\right],
$$

where $(1) G_{1}, \ldots, G_{k}$ are all the distinct predicate variables occurring free in $\varphi$, and (2) $\varphi$ is a formula in which (a) no bound predicate variables occur, and (b) in which no nonlogical constants occur as well, and (c) in which $F^{n}$ 
has no free occurrences, and, finally, (d) in which the identity sign also does not occur.

Every instance of the conceptualist principle (CCP!) is obviously also an instance of the nominalist principle, (CP!), because universal generalizations of instances of axiom schemas are also assumed to be instances of (or otherwise derivable from) those schemas. But, not for every instance of the nominalist principle, (CP!), is also an instance of the conceptualist principle, (CCP!). In an applied instance of (CP!), for example, but not in an applied instance of (CCP!), the formula $\varphi$, which in general will be a first-order formula of an applied theory, may contain occurrences of the identity sign or of any predicate constant of the language of that theory. That is because bound predicate variables, when interpreted substitutionally, have such formulas as their substituends. In constructive conceptualism, on the other hand, bound predicate variables are not interpreted substitutionally but referentially, albeit not in the sense of reference that applies to bound individual variables. In particular, bound predicate variables have 'predicative' concepts as their values in a framework in which it is not assumed that all first-order formulas, and especially not identity formulas, stand for predicative' concepts. Unlike the situation in nominalism with its substitutional interpretation, an open first-order formula does not represent a 'predicative' context in constructive conceptualism merely because it is a first-order formula.

Consider, for example, a theory of membership containing ' $\in$ ' as its only primitive predicate constant and with the following as its principal axiom:

$$
(\forall F)(\exists y)(\forall x)[x \in y \leftrightarrow F(x)]
$$

In nominalism, where predicate quantifiers are interpreted substitutionally, this axiom, which otherwise seems quite plausible as a thesis to the effect that every predicate expression has an extension, leads directly to Russell's paradox. For, by the nominalist principle, (CP!),

$$
(\exists F)(\forall x)[F(x) \leftrightarrow x \notin x],
$$

is provable under such an interpretation, from which it follows that $x \notin x$ can be properly substituted for $F$ in a universal instantiation of (C). In constructive conceptualism, on the other hand, (D) is not an instance of the conceptualist principle, (CCP!), and all that follows by Russell's argument from (C) is the fact that ' $\in$ ' cannot stand for a 'predicative' (relational) concept. That is, instead of the contradiction that results when predicate 
quantifiers are interpreted substitutionally, (C), when taken as an axiom of a theory of membership in constructive conceptualism, only leads to

$$
\neg(\exists R)(\forall x)(\forall y)[R(x, y) \leftrightarrow x \in y] .
$$

Thus, as a plausible thesis to the effect that every 'predicative' concept has an extension, (C) is consistent, not inconsistent, in constructive conceptualism.

On Quine's nominalist strategy, where concepts are precluded in favor of words or expressions, the notion of a 'predicative' context is purely grammatical; for an open formula is 'predicative' in nominalism just in case it contains no bound predicate variables. In constructive conceptualism, the notion of a 'predicative' context is semantical, which means that in addition to being 'predicative' in nominalism's purely grammatical sense, it must also stand for a 'predicative' concept. It is for this reason that the second-order predicative logic of constructive conceptualism must be free of existential presuppositions regarding predicate constants, which is why the binary predicate, ' $\in$ ', in a theory of membership having $(\mathbf{C})$ as an axiom, cannot stand for a value of the bound predicate variables. In general, how we determine which, if any, of the primitive predicate constants of an applied theory stand for a 'predicative' concept depends on the domain of discourse of that theory and how that domain is to be conceptually represented. In particular, those primitive predicates that are to be taken as standing for a 'predicative' concept will be stipulated as doing so in terms of the 'meaning postulates' of that theory, whereas those that are not will usually occur in axioms that determine that fact.

Identity and its role in a logical theory marks another important difference between Quine's nominalist strategy and our conceptual approach. For Quine, identity is definable in any applied theory with finitely many predicate constants in terms of a formula representing indiscernibility with respect to those predicate constants (cp. Quine 1970, pp. 63ff). That is, in any given application (based on finitely many predicate constants, which we may assume to be the 'standard' situation), identity, on Quine's nominalist strategy, is reducible to a first-order formula, which is why the identity sign is allowed to occur in instances of (CP!) under its nominalistic, substitutional interpretation. Such a definition will not suffice in constructive conceptualism, on the other hand, because the first-order formula in question, even were it to stand for a 'predicative' concept, cannot justify the substitutivity of 'identicals' in impredicative contexts, that is, contexts that do not represent a 'predicative' 
context. The identity sign is not eliminable, or otherwise reducible, in constructive conceptualism, in other words, because, on the basis of Leibniz's law, identity must allow for full substitutivity even in impredicative contexts. Thus, whereas,

$$
x=y \leftrightarrow(\forall F)[F(x) \leftrightarrow F(y)],
$$

is provable in standard predicative second-order logic, as based on its nominalistic substitutional interpretation, the right-to-left direction of this same formula is not provable in the nonstandard predicative second-order logic that is validated in constructive conceptualism.

Finally, it is important to see why, although impredicative definitions are precluded in the predicative logic validated in nominalism, they are not precluded in the predicative logic validated in constructive conceptualism. The difference is determined by the role free predicate variables have in each of these frameworks. On Quine's strategy, as already noted, free predicate variables are construed as dummy schema letters, which in an applied theory stand for arbitrary first-order formulas of that theory. This means that the substitution rule,

$$
\text { if } \vdash \psi \text {, then } \vdash \psi\left[\varphi / G\left(x_{1}, \ldots, x_{n}\right)\right] \text {, }
$$

is valid on the substitutional interpretation only when $\varphi$ is 'predicative' in nominalism's purely grammatical sense, i.e., only when no predicate variable has a bound occurrence in $\varphi$. Indeed, the rule must be restricted in this way on Quine's strategy because, otherwise, by taking $\psi$ to be the following instance of (CP!),

$$
(\exists F)\left(\forall x_{1}\right) \ldots\left(\forall x_{n}\right)\left[F\left(x_{1}, \ldots, x_{n}\right) \leftrightarrow G\left(x_{1}, \ldots, x_{n}\right)\right],
$$

we would be able to derive the full, unrestricted impredicative comprehension principle and, thereby, transcend the substitutional interpretation of predicate quantifiers.

In the predicative logic of constructive conceptualism, on the other hand, the above substitution rule is valid for all formulas, regardless whether or not they contain any bound predicate variables. But, unlike the situation in nominalism, the validity of such a rule does not lead to the unrestricted impredicative comprehension principle. In particular, the above instance of (CP!) is not also an instance of (CCP!). This means that the notion of a possible (explicit) definition of a predicate constant is broader in constructive 
conceptualism than it is in nominalism, where only first-order formulas are allowed as definiens. On the basis of the rule of substitution for all formulas, it can be shown that definitions in constructive conceptualism whose definiens contain a bound predicate variable will still be noncreative and will still allow for the eliminability of defined predicate constants (cf. Cocchiarella 1986a, chap. 2, sec. 3). Thus, even though constructive conceptualism validates only a predicative comprehension principle, i.e., a comprehension principle encompassing laws of compositionality that are in accordance with the vicious circle principle, it nevertheless allows for impredicative definitions of predicate constants, that is, of predicate constants that do not stand for values of the bound predicate variables and that cannot therefore be existentially generalized upon. Thus, by refusing to consider the cognitive structures that underlie our use of language, Quine's nominalist strategy leads us to the wrong principles underlying our use of language and away from otherwise acceptable patterns of definition only because they contain bound predicate variables.

\section{Conceptualism Ramified Versus Nominalism Ramified}

There is a way of allowing bound predicate variables to occur in definitions even on Quine's nominalist strategy, which is not to say that such definitions will be impredicative. In particular, a substitutional interpretation of predicate quantifiers can be given not just for standard predicative secondorder logic but for standard ramified second-order logic as well. (Cf. Church (1956, sec. 58) for a formulation of standard ramified second-order logic.) The idea of such an interpretation is that we are to begin with a language $L_{0}$ for standard predicative second-order logic and proceed from there through a potential infinity of languages $L_{1}, \ldots, L_{n}, \ldots(n \in \omega)$, where the predicate variables of $L_{j+1}$ have the formulas of $L_{j}$ as their substituends, and where the predicate quantifiers binding those variables are interpreted substitutionally with respect to their substituends. These languages can be cumulative, but because the formulas of $L_{j}$ may contain bound occurrences of predicate variables, the predicate variables of $L_{j+1}$ must then be different from the predicate variables of $L_{j}$. In this way, definitions occurring in $L_{j+1}$ can contain bound predicate variables in their definiens, but only the bound pred- 
icate variables of $L_{j}$ or of any of the languages preceding $L_{j}$ in the sequence, and none at all from $L_{j+1}$ or languages succeeding $L_{j+1}$ in the sequence.

There is a ramified logic for constructive conceptualism as well, which differs from the standard ramified second-order logic validated by nominalism in essentially the same way that the nonstandard predicative logic described above differs from standard predicative second-order logic. In particular, the logic is free of existential presuppositions regarding predicates, and for that reason there is no need for the different kinds of predicate variables that nominalism requires for each of the languages $L_{j}$. Indeed, because predicate quantifiers are interpreted referentially and not substitutionally, the sort of ramification validated in this framework is based not on a sequence of languages but on a sequence of stages of concept-formation instead, where the concepts formed at one stage become the referents of the concepts formed at the next. The stages are cumulative, accordingly, and what is added at each stage $j+1$ beyond the initial stage of the 'predicative' concepts of an applied theory are concepts whose construction involves a reference to the totality of concepts constructible at the $j t h$ stage. Because these new concepts do not involve or presuppose any reference to their own totality, i.e., because they are formed in accordance with the vicious circle principle, they, too, are considered to be 'predicative' concepts. Thus, instead of the infinite sequence of different kinds of predicate variables required in nominalism, what is formally required in constructive conceptualism is a sequence of different quantifier signs $\forall^{1}, \exists^{1}, \ldots, \forall^{i}, \exists^{i}, \ldots(j \in \omega-\{0\})$, all of which can be affixed to the same predicate variables. The predicate quantifiers $\left(\forall^{j} F\right)$ and $\left(\exists^{j} F\right)$, where $F$ is an $n$-place predicate variable, will then be understood to refer in a given applied theory to all, or to some, respectively, of the $n$-ary 'predicative' concepts that can be formed in that theory at the $j t h$ stage of the potentially infinite sequence of stages of concept-formation in question. But, then, because open formulas representing 'predicative' contexts of later stages will not be substituends of predicate variables bound by quantifiers of an earlier stage, this means that the logic of the quantifiers $\forall^{j}$ and $\exists^{j}$ must be free of existential presuppositions regarding predicate expressions, which is why the comprehension principle for this logic must be closed with respect to all the predicate variables occurring free in the comprehending formula. Thus, as applied at the $j t h$ stage, the ramified conceptualist comprehension principle that is validated in this framework is the following:

$(\mathbf{R C C P} !) \quad\left(\forall^{j} G_{1}\right) \ldots\left(\forall^{j} G_{k}\right)\left(\exists^{j} F\right)\left(\forall x_{1}\right) \ldots\left(\forall x_{n}\right)\left[F\left(x_{1}, \ldots, x_{n}\right) \leftrightarrow \varphi\right]$ 
where: (1) $G_{1}, \ldots, G_{k}$ are all of the predicate variables occurring free in $\varphi$; (2) $F$ is an $n$-place predicate variable not occurring free in $\varphi ;(3) x_{1}, \ldots, x_{n}$ are distinct individual variables; and (4) $\varphi$ is a pure ramified formula, i.e., one in which no nonlogical constants occur and in which (a) the identity sign also does not occur and (b) in which no predicate variable is bound by a quantifier of a stage $>j$ (i.e., for all $i \geq j$, neither $\forall^{j}$ nor $\exists^{j}$ occurs in $\varphi$ ).

By way of a more intuitive explanation of this principle, we should note that a basic assumption of conceptualism, as we understand it here, is that concept-formation proceeds through developmental stages of increasing structural complexity, where the later stages contain reconstructions of the earlier. We assume, moreover, that the psychological mechanism underlying these reconstructions is something like what Jean Piaget, in his genetic epistemology, has described as autoregulation, a mechanism that is driven by an equilibration process that moves us from states of lesser cognitive equilibrium, where our concepts are unable to explain certain aspects of the environment, to states of greater equilibrium, where new conceptual structures give us more explanatory power (cf. Piaget 1977). This same process, at the stage of conceptual development we are concerned with here, is also called reflective abstraction and, involves a projection of previously constructed concepts onto a new plane of thought where they are reorganized under the closure conditions of new laws of concept-formation characteristic of the stage in question. One such pattern of reflective abstraction is precisely what is represented by the ramified comprehension principle and free predicative logic of constructive conceptualism, where each of the successive stages of concept-formation in the ramified hierarchy is generated by a disequilibrium, or conceptual tension, between the predicate expressions that stand for 'predicative' concepts at the preceding stage, as opposed to those that do not. The attempt to overcome this disequilibrium, by rendering more and more such expressions 'predicative' at successive stages, is the motivating force that drives us on from one stage of the ramified hierarchy to the next. In nominalism, on the other hand, where there is no 'free logic' regarding predicate expressions and no difference between grammatical and semantical predicativity, there is also no motivating force that moves us on from one language of the nominalist's ramified hierarchy to the next.

Whatever the motivation for ramification in nominalism, it is clear that what moves us on from one stage of concept-formation to the next in constructive conceptualism is a drive for closure, where all predicates stand for concepts. Such a closure cannot be realized in constructive conceptualism, 
to be sure, where the principal constraint guiding the formation of "predicative' concepts is their being specifiable by conditions that are in accord with the so-called vicious circle principle. But the particular pattern of reflective abstraction that corresponds to this constraint is not all there is to autoregulation and the general process of reflective abstraction, and, in fact, as a pattern that represents a drive for closure, it contains the seeds of its own transcendence to a new plane of thought where such closure is achieved. The general process of constructing new patterns of reflective abstraction is not itself constrained, in other words, by the so-called vicious circle principleat least, not as described by Piaget. In particular, after achieving what Piaget calls the stage of formal operational thought, certain new patterns of concept-formation are realizable, albeit usually only in post-adolescence, that involve an idealized transition to a limit, where impredicative conceptformation becomes possible. Such an idealized transition, in the case of our ramified logic, is conceptually similar to, but ontologically different from, an actual transition to a limit at an infinite stage of concept-formation, that is, a stage that is not only the summation of all of the finite stages of the ramified hierarchy but one that is also closed with respect to itself. Ontologically, of course, there cannot be an infinite stage of concept-formation for concepts as cognitive capacities, but that is not to say that an idealized transition to a limit is conceptually impossible as well. Indeed, in what I have else; called holistic conceptualism, such an idealized transition to a limit is precisely what is assumed to be possible on the basis of the pattern of reflective abstraction represented by (RCCP!*). That is, in holistic conceptualism, the drive for closure upon which the pattern of reflective abstraction of ramified constructive conceptualism is based is finally achieved, albeit only as the result of an idealized transition to a limit and not on the basis of an actual transition. In this way, conceptualism, by means of a mechanism of autoregulation that enables us to construct stronger and more complex logical systems out of weaker ones, is able to validate not only the ramified conceptualist comprehension principle but also the full, unqualified impredicative comprehension principle of standard second-order logic. There is no comparable mechanism in nominalism that can similarly lead to a validation of the impredicative comprehension principle. 


\section{Constructive Conceptual Realism Versus Quine's View of Conceptualism as a Ramified Theory of Classes}

Quine has not always insisted on by-passing conceptualism in favor of nominalism. In fact, in his 1947 paper, 'Logic and the Reification of Universals', where he describes what he takes to be the logical differences between Platonism, conceptualism, and nominalism, Quine actually suggests that from a "tactical" point of view, "conceptualism is no doubt the strongest position of the three" (Quine 1953, p. 129). But, by conceptualism, Quine does not mean what we mean, namely, a theory in which concepts are taken as the values of bound predicate variables; rather, what he means is a ramified theory of classes, and in particular a theory based on standard ramified secondorder logic, but with predicates allowed to occur as abstract singular terms and therefore a theory in which predicate quantifiers are to be interpreted referentially and not substitutionally. Also, by nominalism, Quine means more than a nominalist theory of predication, for, by his lights, nominalism requires that we forswear not just all predicable entities (i.e., universals in the traditional sense) beyond predicates themselves, but all abstract entities of whatever sort or type. This is a different view than is maintained, for example, by Nelson Goodman, according to whom nominalism "does not involve excluding abstract entities ... but requires only that whatever is admitted as an entity at all be construed as an individual", where to construe entities as individuals means that we "take them as values of the variables of lowest type in the system" (Goodman 1956, p. 17). Quine concurs that, in the end, any entity at all that we are committed to should be taken as a value of the bound individual variables, but he also insists that nominalism precludes abstract entities from being among such values.

The issue here is more than terminological because it turns on Quine's insistence that predicate quantifiers can be given a referential interpretation only if predicates are themselves (mis)construed as singular terms and, given his further insistence on extensionality, that predicates, as singular terms, can only denote classes, which then are also the values of the bound predicate variables. Predication in such a framework can only be membership, in other words, in which case we might as well replace predicate variables with individual variables and take classes, the 'universals' or predicable entities of this framework, as values of those variables, thereby arriving finally at 
a first-order theory of classes, to which, we may assume, all other abstract entities (such as numbers, etc.) are reducible. In this way, nominalism as a theory of predication, and in particular as a theory that disallows predicate variables from having any entities (i.e., 'universals' in the traditional sense) as their values, becomes nominalism as a doctrine that forswears abstract entities as the values of the bound individual variables.

Similarly, conceptualism as a theory in which predicate quantifiers are given a referential interpretation becomes a theory in which predicates must be allowed to occur as abstract singular terms, and therefore the objects denoted by predicates as singular terms, which, again, assuming extensionality, can only be classes, must also be the values of the bound predicate variables. Predication is again replaced by membership, and predicate variables and predicate quantifiers are replaced by individual variables and first-order (objectual) quantifiers. In this way, conceptualism as a theory of predication, and in particular as a theory in which bound predicate variables have concepts as their values, becomes a first-order theory of membership in which concepts are reified as classes. Where conceptualism differs from Platonism in this regard is in having its logic based on a predicative, as opposed to an impredicative, comprehension principle, and in particular on the predicative comprehension principle of standard ramified second-order logic, but extended so that predicate letters are allowed to occur as abstract singular terms.

Thus, on Quine's description, conceptualism begins with an infinity of languages $L_{0}, \ldots, L_{n}, \ldots(n \in \omega)$, where the individual variables of $L_{0}$ have only concrete objects as their values. Then, "the first step of reification of classes is to be limited to classes such that membership in any one of them is equivalent to some condition expressible in $L_{0}$; and correspondingly for relations" (Quine 1953, p. 123). Calling these classes and relations objects of order 1, we are to "begin binding predicate letters with the idea that they are to admit objects of order 1 as values" (ibid.), which means that the predicate letters, to which we are to attach the exponent ' 1 ', are allowed to occur as abstract singular terms as well. $L_{1}$ is the language formed by thus extending $L_{0}$. Then, "the next step is to reify all further classes of such kind that membership in any one of them is equivalent to some condition expressible in $L_{1}$; and similarly for relations" (ibid., p. 124). Calling these classes ' $F^{2}$ ', ' $G{ }^{2}$, etc., with the idea that they take objects of order 2 as their values. "Continuing thus to $L_{3}, L_{4}$, and so on, we introduce bound [predicate] variables with ever-increasing exponents, concomitantly admitting increasingly wide ranges 
of classes and relations as values of our variables" (ibid.). Finally, "we can omit the polyadic use of bindable variables; and we can rewrite the residual forms ' $F^{3} x^{\prime}$, ' $G^{2} F^{3}$ ', etc., in the preferred notation ' $x^{0} \in y^{3}$ ', ' $y^{3} \in z^{2}$ ', etc .... There are no restrictions analogous to those of the theory of types: no requirements of consecutiveness, indeed no restrictions on meaningfulness of combinations" (ibid.).

The idea that bound predicate variables can have unsaturated concepts as their values, and therefore that what nominalized predicates denote as abstract singular terms, assuming that they denote at all, cannot be the values of those variables is a notion that Quine never considers, and yet it is a notion that is fundamental to conceptualism as we have described it here. It is also a notion that conceptualism shares with Frege (or really adopted from Frege), who assumed not only that nominalized predicates denote but that what they denote are the extensions of the concepts that those predicates otherwise stand for in their role as predicates. Such an assumption about nominalized predicates goes beyond conceptualism, as we understand it here, but it does not go beyond what might be called conceptual realism. Contrary to Quine's view, in other words, conceptualism not only is not the same as a theory of classes, ramified or otherwise, but it does not of itself even contain a theory of classes. Conceptual realism, on the other hand, does contain such a theory, and constructive conceptual realism in particular contains a ramified theory but not one that is based on standard ramified second-order logic. It is not the standard, but the nonstandard, ramified second-order logic described above that is the framework that should be associated with constructive conceptual realism when nonvacuous nominalized predicates are added to it as abstract singular terms.

We will not go into the details of the formulation of constructive conceptual realism, which is given elsewhere (in Cocchiarella 1986b), except perhaps to note that we now apply the 'stage' quantifiers $\forall^{j}$ and $\exists^{j}$, for all positive integers $j$, not just to predicate variables but to individual variables as well, to which we also continue to affix the absolute quantifiers $\forall$ and $\exists$. (The latter are affixed to predicate variables as well only in holistic conceptualism, realist or otherwise.) Thus, whereas the realist assumption that every $n$-ary concept of each stage $j$ of the ramified hierarchy has an object of that stage as its correlate is formulated as follows,

$$
\left(\forall^{j} F^{n}\right)\left(\exists^{j} x\right)(F=x),
$$

the assumption that every object of stage $j$ is an object simpliciter is formu- 
lated as

$$
\left(\forall^{j} x\right)(\exists y)(x=y) .
$$

Also, because not all objects that are correlates of concepts of later stages will be values of variables bound by quantifiers of earlier stages, the logic of the quantifiers $\forall^{j}$ and $\exists^{j}$, as affixed to individual variables, will be free of existential presuppositions regarding singular terms, just as the logic of the same quantifiers, when affixed to predicate variables, is free of existential presuppositions regarding predicate expressions. Thus, just as the comprehension principle of ramified constructive conceptualism must be closed with respect to the predicate variables occurring free in the comprehending formula, so too, the comprehension principle for constructive conceptual realism must be closed with respect to the individual as well as the predicate variables occurring free in that formula. In terms of $\lambda$-abstracts, the ramified conceptualist comprehension principle for this logic can then be formulated as follows:

$\left(\mathbf{R C C P} !_{\lambda}^{*}\right) \quad\left(\forall^{j} y_{1}\right) \ldots\left(\forall^{j} y_{m}\right)\left(\forall^{j} G_{1}\right) \ldots\left(\forall^{j} G_{k}\right)\left(\exists^{j} F\right)\left(\left[\lambda x_{1} \ldots x_{n} \varphi\right]=F\right)$,

where: (1) $\varphi$ is a formula in which no nonlogical constants occur and in which the identity sign also does not occur; (2) $F$ is an $n$-place predicate variable not occurring free in $\varphi$; (3) for all $i \geq j$, neither $\forall^{j}$ nor $\exists^{j}$ occur in $\varphi$; (4) $G_{1}, \ldots G_{k}$ are all of the distinct predicate variables occurring in $\varphi$; and (5) $y_{1}, \ldots, y_{m}, x_{1}, \ldots, x_{n}$ are all of the distinct individual variables occurring free in $\varphi$.

The assumption that the objects denoted by nominalized predicates are classes, specifically the extensions of the 'predicative' concepts that those predicates otherwise stand for in their role as predicates, can be made explicit in the form of an extensionality axiom. Membership can then be defined for each stage as follows:

$$
x \in_{j} y={ }_{d f}\left(\exists^{j} F\right)[y=F \wedge F(x)] .
$$

But even without the extensionality axiom, it is provable in constructive conceptual realism that every 'predicative' unary concept of any stage $j$ will have an extension in the following sense,

$$
\left(\forall^{j} F\right)(\exists y)(\forall x)\left[x \in_{j} y \leftrightarrow F(x)\right]
$$

which suggests that we might avoid extensionality altogether and assume that concept-correlates are intensional objects instead. Following something 
like Russell's strategy in his "no classes" theory, all talk of classes can be contextually defined in terms of extensional talk about intensional objects, where, by an intensional object, we mean the intension of a concept in the sense of a reification of the truth conditions determined by the different possible applications of that concept. Thus, not only does a class as the extension of a concept have its being in that concept but so does an intensional object as the intension of that concept. It is in this way that conceptual realism is able to explain how, by means of our conceptual abilities, we are able to lay hold upon the intension of a concept, and thereby its extension as well, by starting out from the concept as a cognitive capacity.

Bertrand Russell, in his own intensional ramified logic, thought that he had to assume what he called the reducibility axiom, which in our formulation is the thesis that every concept of any stage $j$ is coextensive with a concept of the first stage, or, formally,

$$
\left(\forall^{j} G\right)\left(\exists^{1} F\right)\left(\forall x_{1}\right) \ldots\left(\forall x_{n}\right)\left[F\left(x_{1}, \ldots, x_{n}\right) \leftrightarrow G\left(x_{1}, \ldots, x_{n}\right)\right],
$$

in order to give an adequate account of classes in terms of intensional entities. Such an axiom, as Quine has observed, does hot accord well with the constructive approach of Russell's ramified type theory, and certainly it cannot be validated in constructive conceptualism. But Quine seems to go too far in claiming that "the axiom of reducibility implies the superfluousness of the very distinctions that gave it substance" (Quine 1963, p. 253). For, unless we assume the extensionality axiom, the reducibility axiom does not imply

$$
\left(\forall^{j} G\right)\left(\exists^{1} F\right)(G=F)
$$

in the ramified logic of constructive conceptual realism. The reducibility axiom does have some other interesting consequences in this framework, however, such as that identity cannot be represented by any relational 'predicative' concept of any stage $j$, that is, formally, it implies

$$
\neg\left(\exists^{j} R\right)(\forall x)(\forall y)[R(x, y) \leftrightarrow x=y] .
$$

This shows that Russell's claim that, on the basis of the reducibility axiom, identity can be adequately analyzed as indiscernibility with respect to all the 'predicative' concepts of the first stage is false. Indeed, where indiscernibility in this restricted sense is defined as follows,

$$
x \equiv_{1} y=_{d f}\left(\forall^{1} F\right)[F(x) \leftrightarrow F(y)],
$$


it can be proved on the basis of the reducibility axiom that there are nonidentical objects (concept-correlates in particular) that are indiscernible in this sense. That is, instead of showing that identity can be analyzed as indiscernibility with respect to the 'predicative' concepts of the first stage, the reducibility axiom actually implies the opposite in having

$$
(\exists x)(\exists y)\left(x \equiv_{1} y \wedge x \neq y\right)
$$

as one of its consequences. ${ }^{25}$

It is noteworthy, incidentally, that each of these consequences of the reducibility axiom has already been noted and discussed in somewhat different terms in the context the impredicative logic $\boldsymbol{\lambda} \mathbf{T}^{*}$ mentioned in Section 5. For if we assume that the identity sign does not occur in any of the $\lambda$-abstracts of the ramified logic of constructive conceptual realism (i.e., as well as in none of the instances of $\left(\mathbf{R C C P} !_{\lambda}^{*}\right)$, from which it is already barred) then, by replacing each occurrence of the 'stage' quantifiers $\forall^{j}$ and $\exists^{j}$ by the absolute quantifiers $\forall$ and $\exists$, it can be shown that every theorem of the ramified logic in question, plus the reducibility axiom as well, is a theorem of $\boldsymbol{\lambda} \mathbf{T}^{*}$, which, as already noted, has an absolute consistency proof. If we allow the identity sign to occur in $\lambda$-abstracts, as is only proper in conceptualism, a similar relative consistency proof can be given with respect to the system $\mathbf{T}_{\lambda}^{*}$, which has even the logic of the absolute quantifiers $\forall$ and $\exists$ free of existential presuppositions regarding singular terms. ${ }^{26}$

What makes the reducibility axiom useful in a ramified logic is that it enables us to prove an extensional impredicative comprehension principle, which can be used in place of an extensionality axiom in the development of a contextually defined theory of classes as the extensions of concepts. But this does not make the reducibility axiom any the less implausible as a thesis about 'predicative' concepts. In fact, given its impredicative content, the reducibility axiom is really a poor compromise between holistic conceptualism with its validation of an impredicative comprehension principle and a strict version of constructive conceptualism that refuses to acknowledge any psychological mechanism (such as Piaget's autoregulation) that would enable us

\footnotetext{
${ }^{25}$ For a proof of these and other consequences of the reducibility axiom in the ramified logic of constructive conceptual realism, see Cocchiarella (1986b, sec. 6).

${ }^{26}$ I am grateful to Max Freund, for pointing out that allowing the identity sign to occur in $\lambda$-abstracts means that the consistency proof for the ram constructive conceptual realism must be relativized not to $\boldsymbol{\lambda} \mathbf{T}^{*}$, as I originally claimed, but to $\mathbf{T}_{\lambda}^{*}$ instead.
} 
to construct impredicative concepts. If an impredicative comprehension principle is to be validated at all, in other words, then it is conceptually clearer that it be validated on the basis of an idealized transition to a limit and a pattern of reflective abstraction by means of which impredicative conceptformation becomes possible. Such a transition does not nullify the ramified hierarchy of 'predicative' concepts, and in fact were we to add an epistemic operator $\square^{k}$, which can be informally read as, 'It is constructively knowable that', then one important difference between the predicative comprehension principle (RCCP $!_{\lambda}^{*}$ ) of constructive conceptualism and the impredicative comprehension principle, $\left(\mathbf{C P}_{\lambda}^{*}\right)$, of holistic conceptualism is that $\square^{k}$ can be validly inserted only after the existential posit $\left(\exists^{j} F\right)$ in (RCCP $\left.{ }_{\lambda}^{*}\right)$, but, not after the existential posit $(\exists F)$ in $\left(\mathbf{C P}_{\lambda}^{*}\right)$. It is not just that there is a 'predicative' concept that is represented by any formula fulfilling the 'predicative' conditions of (RCCP! $\left.{ }_{\lambda}^{*}\right)$ but, rather, that there is a 'predicative' concept for which it is constructively knowable that it is represented by that formula. No such constructive knowledge can be similarly assumed regarding the concepts posited in the impredicative comprehension principle.

The transition to holistic conceptualism and the validation of an impredicative comprehension principle regarding concept-formation does not mean that a similar transition to a realist assumption about nominalized predicates is also thereby validated. Indeed, because some nominalized predicates, such as the $\lambda$-abstract for the Russell predicate, $[\lambda x(\exists G)(x=G \wedge \neg G(x))]$, will necessarily fail to denote any object at all, despite the fact that the predicate itself, in its role as a predicate, will stand for an impredicative concept, the logic of the absolute quantifiers $\forall$ and $\exists$, when affixed to individual variables, must be free of existential presuppositions regarding singular terms, which means that no realist theses beyond the original realist assumptions of constructive conceptual realism will follow in this extended framework. This allows for an intermediate position between constructive conceptual realism and one or another version of a more thoroughgoing holistic conceptual realism, where, on the intermediate position, it is not the specification of impredicative concepts that is objectionable, but the assumption of objects, e.g., classes, corresponding to such concepts. By covering all that one might want to do with the reducibility axiom, and more, such an intermediate position shows why, on at least our version of conceptualism, where there is a difference between the positing of concepts and the positing of classes as the extensions of those concepts, Quine is wrong to claim that the reducibility axiom "has the effect of reinstating the whole platonistic logic of classes" 
(Quine 1953, p. 127).

\section{Holistic Conceptual Realism Versus Quine's Class Platonism}

Despite his statement that conceptualism, when compared with nominalism and Platonism, is "the strongest position of the three", Quine, in the end, opts for Platonism, which for him means a full, impredicative theory of classes. Conceptualism, as a ramified theory of classes, is too weak a framework in which to develop the mathematics (such as the theory of real numbers) that is needed in empirical science. In addition, the idea of classes as "conceptual in nature and created by man" (ibid., p. 123) and the idea in particular of the classes of the ramified hierarchy being generated by a "progressive creation" is at best a metaphor according to Quine, for whom "it would be a mistake to suppose that this metaphor really accounts for the classes or explains them away" (ibid., p. 127). Thus, on Quine's view, "the conceptualist and the platonist are alike: they both assume universals, classes, irreducibly as values of their bound variables" (ibid.), in which case we might as well join the Platonist and assume an impredicative theory of classes adequate for the purposes of empirical science.

Quine is right in noting that a ramified predicative logic is too weak for the purposes of the theory of real numbers. But the issue here is not just one of ontology, that is, of whether or not a ramified predicative logic can provide an adequate ontological account of the theory of real numbers as mathematical objects. What is inadequate about the logic of constructive conceptual realism in an even more fundamental way is that it cannot provide an account of the kind of impredicative concept-formation that is necessary for the development and use of the theory of real numbers, and which, as a matter of cultural history, we have in fact achieved since the nineteenth century. The concept of a least upper bound, for example, or of the limit of a converging sequence of rational numbers, is an impredicative concept that was not acquired by the mathematical community until a little more than a century ago; and although, in our own time, it is not usually a part of a person's conceptual repertoire until post-adolescence, nevertheless, with

proper training and conceptual development, it is a concept that most of us can come to acquire as a cognitive capacity. Yet, notwithstanding these 
facts of cultural history and conceptual development, it is also a concept that cannot be accounted for from within the framework of constructive conceptualism. The constraints of the vicious circle principle, at least in the way they apply to concept-formation, simply do not conform to the facts of conceptual development in an age of advanced scientific knowledge.

This does not mean that constructive conceptualism is wrong in its account of concept-formation as regards a certain stage of conceptual development. In fact, to the contrary, it is an essential precursor, both historically and ontogenetically, to the kind of reflective abstraction that leads to impredicative concept-formation. ${ }^{27}$ What is wrong is claiming, as Russell and Quine do from the perspective of their different versions of Platonism, that the whole point of a ramified predicative logic is avoidance of Russell's paradox. Certainly, that was not why Hermann Weyl adopted a predicative logic as the basis of his constructive mathematics; nor was it the reason why Henri Poincaré originally proposed his vicious circle principle, which Russell accepted and adapted to his own purposes (cf. Weyl 1918 and Poincaré 1906). In fact, as indicated in the preceding section, the logical structure of the kinds of concepts that are represented in constructive conceptualism, when viewed from within the framework of holistic conceptualism, provides the basis for just the kind of epistemic distinction that Weyl and Poincaré had in mind, namely, the distinction between knowledge of abstract objects that is constructive, as opposed to knowledge that is not, and in particular, knowledge that is based on impredicative concepts.

In regard to ontology, or any realist assumptions that can be logically associated with impredicative concept-formation, it should again be emphasized that the transition from constructive conceptualism to holistic conceptualism enables us to validate only the impredicative comprehension principle

\footnotetext{
${ }^{27}$ In Inhelder and Piaget (1958) it is pointed out that the maturation of the human nervous system in adolescence "can do no more than determine the totality of possibilities and impossibilities [of concept-formation] at a given stage" and that "a particular social environment remains indispensable for the realization of these possibilities" (p. 337). That is, conceptual development "can be accelerated or retarded as a function of cultural and educational conditions," which is why "the history of formal structures is linked to the evolution of culture and collective representations as well as their ontogenetic history" (ibid.). That is also why, in regard to our present context, even the post-adolescents of primitive societies do not appear to have any ímpredicative concepts as part of their repertoire of cognitive abilities.
} 
for concept-formation, that is, the principle,

$$
\left(\mathbf{C P}_{\lambda}^{*}\right) \quad(\exists F)\left(\left[\lambda x_{1} \ldots x_{n} \varphi\right]=F\right),
$$

where $\varphi$ is any formula of the extended grammar in which the $n$-place predicate variable $F$ does not occur free. What it does not do is enable us to also validate an impredicative theory of classes, or intensional objects, as the correlates of concepts. Of course, we do still have the realist assumption of constructive conceptual realism, which with the epistemic operator $\square^{k}$ can now be formulated as,

$$
\left(\forall^{j} F^{n}\right)\left(\exists^{j} x\right) \square^{k}(F=x) .
$$

But beyond the ramified classes, or intensional objects, that are thus assumed, nothing can be proved about which, if any, of the concepts specified in $\left(\mathbf{C P}_{\lambda}^{*}\right)$ also have objects corresponding to them.

What can be proved negatively, on the other hand, is that not every concept of the extended framework can have an object as its correlate and, in particular, that there can be no such object corresponding to the concept that the Russell predicate stands for. That is, even though

$$
(\exists F)([\lambda x(\exists G)(x=G \wedge \neg G(x))]=F)
$$

is provable in the extended system, so is

$$
\neg(\exists y)([\lambda x(\exists G)(x=G \wedge \neg G(x))]=y)
$$

The result of extending constructive conceptual realism so as to allow for impredicative concept-formation takes us only to the intermediate framework noted at the end of the last section, in other words, i.e., the framework intermediate between constructive conceptual realism and a more thoroughgoing holistic conceptual realism. Such an intermediate position is interesting and useful in emphasizing the distinction between conceptualism and realism, i.e., between concept-formation and ontology, but in the end it leaves us without a logical foundation for an impredicative theory of classes other than something like Quine's version of Platonism.

One might well assume that the ontology of the abstract objects of conceptual realism, be it of a constructive or a holistic conceptual realism, is a form of Platonism, in which case we might as well call the ontological version of conceptual realism in question conceptual Platonism. This is not the 
only way to interpret the ontology of conceptual realism, as we shall see; but, even if we were to accept this interpretation, there is still an important difference between the indirect Platonism thus assumed and the direct, or simple, Platonism of a theory of classes such as Quine intends. Indeed, from the point of view of conceptualism or, rather, conceptual realism, the problem with a direct Platonism (whether as an impredicative theory of classes, as in Quine's version, or, as an impredicative theory of properties and relations, as in Russell's and Frege's versions) is that it fails to explain how it is possible to have knowledge of the independently real abstract entities that it assumes, if all knowledge must be grounded in psychological states and processes that do not have such abstract entities as constituents. If the mind and epistemology are to be 'naturalized', as Quine himself has argued, and as they are assumed to be in conceptualism, then the problem of how our knowledge of the abstract entities assumed in Platonism can be possible seems insuperable - unless, that is, all our knowledge of abstract entities can be explained in terms of concepts as cognitive capacities, which in fact is just what is claimed in conceptual realism. Indeed, according to conceptual realism, whether Platonist or otherwise, we are able to have knowledge of abstract objects at all only because they are the correlates (e.g., extensions or intensions) of concepts, which is to say that we are conceptually able to lay hold upon such objects only by starting out from the concepts whose correlates they are.

Quine agrees with conceptual realism, incidentally, about how we first arrive at the notion of a class: namely, that we do so through the process of nominalization, that is, the process by which predicate expressions are transformed into abstract singular terms (cf. Quine 1974, chap. III). But, instead of basing his account on concepts as the cognitive capacities underlying our use of predicates, Quine adopts the nominalist strategy described earlier where predicate quantifiers, at least initially, are interpreted substitutionally. Once we learn how to nominalize predicates and begin using them as abstract singular terms, according to Quine, the nominalism we began with is given over to a Platonism, where nominalized predicates, in accordance with the assumption of extensionality, are taken as denoting classes, which now can be specified even impredicatively as the values of bound predicate variables. Finally, predication is replaced by membership and nominalized predicates and predicate quantifiers are given over to individual variables and objectual quantifiers in a first-order 'set' theory in which classes, as Platonic entities, can be talked about independently of any predicates whose 
extensions they might or might not be. Thus, by adopting a nominalist strategy and by-passing concepts, Quine moves from his basically correct insight about how nominalization is the key to our understanding of abstract objects which, epistemically, should lead at most to an indirect Platonism in which the role of nominalized predicates is distinguished from that of the predicates themselves - to his direct Platonism of set theory as an impredicative theory of classes.

As a version of conceptual realism, which in general maintains that all abstract objects, be they classes, numbers, or intensional objects, are conceptcorrelates, conceptual Platonism is an indirect and not a direct Platonism. For as concept-correlates, all abstract objects have their being, at least in an epistemological sense, in the concepts whose correlates they are. This means not only that our representation of abstract objects must be seen as the result of the process of nominalization, that is, of transforming predicate expressions into abstract singular terms, whereby we purport to denote the contents or extensions of the concepts that those predicates otherwise stand for in their role as predicates, but, in addition, that such nominalization is not to be explained away by replacing a second-order logic of predication with a first-order theory of membership (or even, for that matter, with a firstorder theory of exemplification). Thus, even though abstract entities may be assumed to exist in a realm that transcends space, time and causality, and in that sense 'preexist' the evolution of consciousness and the cognitive capacities that we exercise in thought and our use of language, nevertheless, from an epistemological point of view, no such entity can be assumed to exist otherwise than as the correlate of a concept. Quine is right in claiming, at least as far as conceptual Platonism is concerned, that classes have the same kind of reality for the conceptualist as they do for the Platonist; but by ignoring concepts and predication and describing conceptualism as a theory of. classes and membership simpliciter, he fails to see the essential epistemological role that concepts have in explaining how our knowledge of classes in the logical sense is even possible at all.

Conceptual Platonism is not the only way to interpret the ontology of conceptual realism, however. There is an alternative interpretation, for example, according to which all abstract objects are in some sense 'man-made', at least in their origin, despite also having a certain degree of autonomy. ${ }^{28}$

\footnotetext{
${ }^{28}$ Cf. Popper and Eccles (1977, chap. P2) for a description of such a view of abstract objects as the denizens of what is there called "world 3". It should perhaps be noted that
} 
They are, as it were, evolutionary products of language and culture, by which we mean that they depend essentially on language and culture for their existence. But, notwithstanding that dependence, they are also an essential part of the means whereby further cultural development - that is, cultural evolution beyond the point of their 'creation'-becomes possible. As cultural products, their existence is primarily the result of the development and use in language of the process of nominalization, i.e., of the process whereby predicate expressions are transformed into abstract singular terms. It was through the institutionalization of this process that abstract entities achieved a certain autonomy and, in time, became reified as objects. Thus, it is not just a matter of how each of us first learns how to talk about classes in today's culture, although that is important as well if we assume what Piaget calls "the fundamental hypothesis of genetic epistemology," namely, that there is a parallel between the ontogenetic and the historical development of scientific knowledge (Piaget 1970, p. 13). Rather, it is a matter of how, over a span of time, through the evolution of the use of nominalized predicates as abstract singular terms, humanity first came to talk about abstract objects at all, and of how, in particular, it came to talk about classes as the extensions of the concepts underlying its use of predicates. Abstract objects do not exist in a Platonic realm outside of space, time, and causality, on this interpretation, but are in fact 'man-made' objects, the result, in effect, of an ontological projection inherent in the development and institutionalization in language of the process of nominalization. Accordingly, on this interpretation, Quine is wrong in claiming that the view of classes as "conceptual in nature and created by man" is only a metaphor and that classes must have the same kind of reality for the conceptualist as they do for the Platonist.

On this interpretation of conceptual realism, what is reified, or projected into the domain of objects, are the contents of our concepts as cognitive capacities, where, by the content of a concept, we mean the truth conditions determined by the different possible applications of that concept, i.e., the conditions under which objects can be said to fall under that concept. If it is assumed that truth conditions can be given only an extensional description, then a class, as the extension of a concept, might well be regarded as the result of reifying the truth conditions determined by that concept, in which

although our view of abstract objects supports the Popper-Eccles interactionist theory of mind, it does not also depend on that theory. 
case we might also assume an axiom of extensionality for our logic. But if, as seems more natural, it is assumed that the truth conditions determined by our concepts can be given an intensional description as well, then abstract objects will have whatever form or degree of intensionality that those truth conditions will be assumed to have, in which case an axiom of extensionality, and perhaps even an axiom of intensionality, will not be warranted. Of course, in some cases, a concept, such as that represented by the Russell predicate, $[\lambda x(\exists G)(x=G \wedge \neg G(x))]$, will determine truth conditions that, on pain of contradiction, cannot be reified, and so the concept itself must be left as having no object as its projected correlate. That is, because the truth conditions determined by an impredicative concept may presuppose the reification of the truth conditions of one or more logically-related concepts, it may well turn out that those truth conditions cannot in turn be reified. Abstract objects are not 'created' at will but can be assumed to exist only in accordance with certain constraints imposed on the use of language, including, in particular, constraints regarding consistency.

We cannot assume, accordingly, that all of the concepts specified in the impredicative comprehension principle, $\left(\mathbf{C P}_{\lambda}^{*}\right)$, have intensions, or extensions, corresponding to them as objects. But that does not mean that there is no principled way by which to assume that most do. One such way, for example, is given by the system $\mathbf{H S T} \mathbf{T}_{\lambda}^{*}$, already described in Section 4, in terms of which the ultimate classes of Quine's 'set' theory ML are better explained as concepts, which now can be understood as the concepts of holistic conceptual realism or, rather, of one version of such. The axiom schema of $\mathbf{H S T}_{\lambda}^{*}$ that describes in a principled way sufficient conditions for positing objects as the correlates of concepts is called $\left(\exists / \mathbf{H S C P}_{\lambda}^{*}\right)$. In this schema, the two principal constraints for positing an object as the correlate of a concept, as represented by a $\lambda$-abstract, $\left[\lambda x_{1} \ldots x_{n} \varphi\right]$, are $(1)$ that the $\lambda$-abstract $\varphi$ must be restricted so as to refer only to concepts that have a conceptcorrelate, i.e., concepts whose truth conditions can be reified. ${ }^{29}$ If the truth conditions determined by an impredicative concept are homogeneously stratified, then those conditions can be reified as an object if the truth conditions determined by any concept presupposed by that concept can also be reified. Thus, we return to the idea of stratification as a fundamental feature not of concept-formation but of the conditions sufficient for reifying the truth

\footnotetext{
${ }^{29} \mathrm{~A}$ detailed, formal description of the axiom scheme $\left(\exists / \mathbf{H S C P}_{\lambda}^{*}\right)$ can be found in Cocchiarella (1986a, p. 229, and 1987, pp. 106ff.).
} 
conditions of our concepts as cognitive capacities, which in turn is the basis of an impredicative theory of classes.

Another principled way by which to assume that most of the concepts specified in the impredicative comprehension principle, $\left(\mathbf{C P}_{\lambda}^{*}\right)$, have an intension or extension corresponding to them as an object is given in the system $\mathbf{T}_{\lambda}^{*}$, which, as explained in Section 5 , is the most natural way of developing the system $\mathbf{T}_{0}^{*}$ to an impredicative logic having $\left(\mathbf{C} \mathbf{P}_{\lambda}^{*}\right)$ as its comprehension principle. $\mathbf{T}_{0}^{*}$, it will be remembered, represents the original logical context (i.e., the second-order logic with nominalized predicates as abstract singular terms) that Quine claimed was inconsistent by Russell's paradox. The simplest extension of $\mathbf{T}_{0}^{*}$ to an impredicative logic is the system $\boldsymbol{\lambda} \mathbf{T}^{*}$, which differs from $\mathbf{T}_{\lambda}^{*}$ in containing a first-order logic that is not free of existential presuppositions for singular terms, and which avoids Russell's paradox by disallowing the identity sign from occurring in $\lambda$-abstracts. The latter exclusion is inappropriate in holistic conceptualism, however, where impredicative concepts such as that represented by the Russell predicate, $[\lambda x(\exists G)(x=G \wedge \neg G(x))]$, are also recognized as cognitive capacities (which can be acquired at least in post-adolescence and in a suitable cultural environment). Allowing the identity sign to occur in $\lambda$-abstracts brings us back to Russell's paradox, however, which, as already explained, can be avoided by taking nominalized predicates to be free of existential presuppositions as abstract singular terms. The result of making these changes to $\boldsymbol{\lambda T}^{*}$ is the system $\mathbf{T}_{\lambda}^{*}$, which is why we say that $\mathbf{T}_{\lambda}^{*}$ is the most natural way of developing $\mathbf{T}_{0}^{*}$ into a system containing the full, impredicative comprehension principle, $\left(\mathbf{C P}_{\lambda}^{*}\right)$ where even the identity sign is allowed to occur in $\lambda$-abstracts.

The logical context that is represented by $\mathbf{T}_{0}^{*}$ is also described by Quine in 'Logic and the Reification of Universals', incidentally, where it is again erroneously claimed to be inconsistent. Indeed, it is on the basis of that claim that the logical context represented by $\mathbf{T}_{0}^{*}$ is changed, or rather modified, by Quine and developed into the ramified theory of classes, that he associates with conceptualism. Of course, as we have already noted, the idea that a ramified predicative logic for constructive conceptual realism is motivated by a response to Russell's paradox is not correct. Nevertheless, there is some irony in the fact that not only is $\mathbf{T}_{0}^{*}$ consistent, but that its most natural development into an impredicative logic, i.e., into the system $\mathbf{T}_{\lambda}^{*}$, can also be seen as a natural way of extending the ramified predicative logic of constructive conceptual realism into a more thoroughgoing holistic conceptual realism. This can be seen by noting that if we begin with the ramified concep- 
tualist comprehension principle, $\left(\mathbf{R C C P} !_{\lambda}^{*}\right)$, as described in Section 7 , and attempt to extend it to an impredicative principle, where the logic of predicate quantifiers is no longer free of existential presuppositions, then the only constraint imposed by $\left(\mathbf{R C C P} !_{\lambda}^{*}\right)$ that remains is that the identity sign is not to occur in the $\lambda$-abstract in question. That is, the natural way of extending (RCCP $\left.!_{\lambda}^{*}\right)$ to an impredicative principle results in the comprehension principle for $\boldsymbol{\lambda} \mathbf{T}^{*}$, which, except for the exclusion of the identity sign from $\lambda$-abstracts, is the same as $\left(\mathbf{C P}_{\lambda}^{*}\right)$. As a thesis about concept-formation, that exclusion, as we have said, is unacceptable in holistic conceptualism. But, as a thesis describing a sufficient condition for positing objects as the correlates of the concepts specified in $\left(\mathbf{C P}_{\lambda}^{*}\right)$ and, in particular, a thesis that sees identity as the real culprit in generating Russell's paradox, then the principle in question, which I have elsewhere called $\left(\exists / \mathbf{C P}_{\lambda}^{* *}\right)$, might well be acceptable on the basis of a doctrine about identity as a transcendental notion. Thus, it is not the system $\boldsymbol{\lambda} \mathbf{T}^{*}$ to which we should turn as the natural way to extend the ramified predicative logic of constructive conceptual realism into a holistic conceptual realism but the system $\mathbf{T}_{\lambda}^{*}$ instead, which contains the axiom schema $\left(\exists / \mathbf{C P}_{\lambda}^{* *}\right)$.

As in the corresponding axiom schema of $\mathbf{H S T}_{\lambda}^{*}$ there are two primary constraints described in $\left(\exists / \mathbf{C P}_{\lambda}^{* *}\right)$ for positing an object as the correlate of a concept as specified in $\left(\exists / \mathbf{C P}_{\lambda}^{* *}\right)$ by means of a $\lambda$-abstract. These are: (1) that all of the predicate quantifiers occurring in the $\lambda$-abstract are restricted in such a way that they refer only to concepts that have conceptcorrelates, i.e., concepts whose truth conditions can be reified; and (2) that the identity sign does not occur in that $\lambda$-abstract at all. In other words, if the truth conditions determined by any concept presupposed by an impredicative concept can be reified, then so can the truth conditions determined by that concept - as long as those conditions do not turn in any way on the concept of identity (which in $\mathbf{T}_{\lambda}^{*}$ is a transcendental notion applicable to concepts as well as to objects).

We thus have two versions of holistic conceptual realism, namely, $\mathbf{H S T}_{\lambda}^{*}$ and $\mathbf{T}_{\lambda}^{*}$, within which we can embed the ramified predicative logic of constructive conceptual realism. Of course, the epistemic operator, $\square^{k}$, will not occur in the existential posits of $\left(\exists / \mathbf{H S C P} \mathbf{P}_{\lambda}^{*}\right)$ or $\left(\exists / \mathbf{C P}_{\lambda}^{* *}\right)$ the way it occurs in the existential posits of constructive conceptual realism. For the abstract objects that are posited in these schemas are in general not posited as the correlates of 'predicative', or constructive, concepts. Both versions contain, either directly or indirectly (depending on one's view of the extensionality 
axiom), an impredicative theory of classes, one corresponding to Quine's 'set' theory ML, and the other corresponding to a natural development of a logical context that Quine incorrectly described as inconsistent. Both provide in this way the basis of a modified account of logicism, founded not on a direct Platonism but at most on indirect Platonism, and perhaps even on a non-Platonistic account of abstract objects as products of cultural evolution. Neither framework alone, for reasons already indicated, will suffice as a foundation for classical mathematics without further posits regarding the reification of the truth conditions determined by certain concepts, such as the concept of a natural number. For conceptualism, however, the important point is not so much the ontology of classical mathematics as the analysis of its concepts, i.e., the representation of those concepts in terms of logical syntax alone. It is in this way, as we have said, that we see where logic leaves off and mathematics begins, and not in Quine's way of taking logic to consist only of elementary, first-order logic and mathematics to consist of a first-order 'set' theory, with or without ultimate classes.

\section{References}

[1] Bochenski. I. M.: 1961, A History of Formal Logic, University of Notre Dame Press, Notre Dame.

[2] Bochenski, I. M., A. Church, and N. Goodman: 1956, The Problem of Universals, University of Notre Dame Press, Notre Dame.

[3] Church, A.: 1956, Introduction to Mathematical Logic, Princeton University Press, Princeton.

[4] Cocchiarella, N.B.: 1973, 'Whither Russell's Paradox of Predication?', in Munitz, 1973, pp. 133-58.

[5] Cocchiarella, N.B.: 1976, 'A Note on the Definition of Identity in Quine's NF', Zeitschr. f. Math. Logik u. Grundlagen d. Math. 22, 195-97.

[6] Cocchiarella, N.B.: 1980, 'Nominalism and Conceptualism as Predicative Second-Order Theories of Predication', Notre Dame Journal of Formal Logic 21, 481-500. 
[7] Cocchiarella, N.B.: 1980, 'The Development of the Theory of Logical Types and the Notion of a Logical Subject in Russell's Early Philosophy', Synthese 45, 71-115 [reprinted in Cocchiarella 1987].

[8] Cocchiarella, N.B.: 1985a, 'Two $\lambda$-Extensions of the Theory of Homogeneous Simple Types', Notre Dame Journal of Formal Logic 26, 377-407.

[9] Cocchiarella, N.B.: 1985b, 'Frege's Double-Correlation Thesis and Quine's Set Theories NF and ML', Journal of Philosophical Logic 14, 1-39 [reprinted in Cocchiarella 1987].

[10] Cocchiarella, N.B.: 1986a, Logical Investigations of Predication Theory and the Problem of Universals, Bibliopolis Press, Naples.

[11] Cocchiarella, N.B.: 1989, 'Conceptualism, Ramified Logic, and Nominalized Predicates', Topoi 5, 75-87.

[12] Cocchiarella, N.B.: 1986c, 'Frege, Russell, and Logicism: A Logical Reconstruction', in Haaparanta and Hintikka 1986 [reprinted in Cocchiarella 1987].

[13] Cocchiarella, N.B.: 1987, Logical Studies in Early Analytic Philosophy, Ohio State University Press, Columbus.

[14] Cocchiarella, N.B.: 1989, 'Conceptualism, Realism, and Intensional Logic,', Topoi 8, 15-34.

[15] Fränkel, A., Y. Bar-Hillel, and A. Levy: 1973, Foundations of Set Theory, North-Holland Pub. Co., Amsterdam.

[16] Frege, G.: 1910, 'Letter to Jourdain', in Bochenski 1961.

[17] Frege, G.: 1952, Translations from the Philosophical Writings of Gottlob Frege, ed. by P. Geach and M. Black, Blackwell, Oxford.

[18] Frege, G.: 1979, Posthumous Writings, ed. by H. Hermes, F. Kambartel and F. Kaulbach, Blackwell, Oxford.

[19] Frege, G.: 1980, Philosophical and Mathematical Correspondence, ed. by G. Gottfried, H. Hermes, F. Kambartel, C. Thiel and A. Veraart, University of Chicago Press, Chicago. 
[20] Goodman, N.: 1956, 'A World of Individuals', in Bochenski et al. 1956.

[21] Haaparanta, L. and J. Hintikka (eds.): 1986, Frege Synthesized, D. Reidel, Dordrecht.

[22] Hahn, L.E. and P.A. Schilpp: 1986, The Philosophy of W.V. Quine, Open Court, La Salle.

[23] Inhelder, B. and J. Piaget: 1958, The Growth of Logical Thinking From Childhood to Adolescence, Basic Books, New York.

[24] Munitz, M.K.: 1973, Logic and Ontology, New York University Press, New York.

[25] Parsons, C.: 1971, 'A Plea for Substitutional Quantification', The Journal of Philosophy, 68, 231-37.

[26] Piaget, J.: 1970, Genetic Epistemology, Columbia University Press, New York.

[27] Piaget, J.: 1977, The Development of Thought, Viking Press, New York.

[28] Poincaré, H.: 1906, 'Les Mathematiques et la Logique', Revue de Metaphysic Moral 14, 294-317.

[29] Popper, K. and J. Eccles: 1977, The Self and Its Brain, Routledge and Kegan Paul.

[30] Quine, W.V.: 1947, 'On Universals', Journal of Symbolic Logic 12, 7484.

[31] Quine, W.V.: 1951, 'Semantics and Abstract Objects', Proc. of the American Academy of Arts and Sciences 80, 90-96.

[32] Quine, W.V.: 1953, From A Logical Point of View, Harvard University Press, Cambridge, MA.

[33] Quine, W.V.: 1963, Set Theory and Its Logic, Harvard University Press, Cambridge, MA.

[34] Quine, W.V.: 1966, The Ways of Paradox, Random House, New York. 
[35] Quine, W.V.: 1970, Philosophy of Logic, Prentice-Hall, Englewood Cliffs.

[36] Quine, W.V.: 1974, The Roots of Reference, Open Court, La Salle.

[37] Quine, W.V.: 1981, Theories and Things, Harvard University Press, Cambridge.

[38] Rosser, J.B.: 1952, 'The Axiom of Infinity in Quine's New Foundations', Journal of Symbolic Logic 17, 238-42.

[39] Russell, B.: 1937, Principles of Mathematics, 2d edn., W.W. Norton \& Co., New York.

[40] Specker, E.: 1953, 'The Axiom of Choice in Quine's New Foundations for Math Logic', Proc. of the Nat. Academy of Sciences 39, 972-75.

[41] Weyl, H.: 1918, Das Kontinuum, De Gruyter, Leipzig. 